\title{
In This World
}

Medienpädagogische Filmanalyse

\section{Jessica Bauer \\ Josefin Kühn \\ Theresia Ertmer}

\section{Zusammenfassung}

Im Rahmen des leitenden Themas „Kinder auf der Flucht" soll der Film "In This World" als ein geeignetes Medium dienen, eine Filmanalyse unter Betrachtung medienpädagogischer Mittel vorzunehmen. Die mehrfach prämierte Semi-Dokumentation vermag die Realität unserer politischen Welt so mit Fiktion und Inszenierung zu verbinden, dass eine klare Trennung vom Zuschauer nicht mehr eindeutig auszumachen ist. Die dadurch überzeugende Darstellung der globalen Wirklichkeit bezieht sich auf eine Problematik, die trotz gesellschaftlicher Kritik oft unausgesprochen bleibt: vom Krieg und dessen Folgen, die in erster Linie die Unschuldigen oder Unbeteiligten solcher Auseinandersetzungen betreffen.

\section{Kontakt}

Theresia Ertmer theresia.ertmer@posteo.de 


\section{Inhalt}

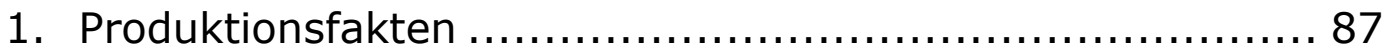

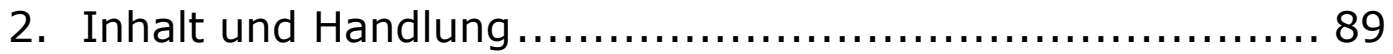

3. Sozialhistorische Analyse ................................. 91

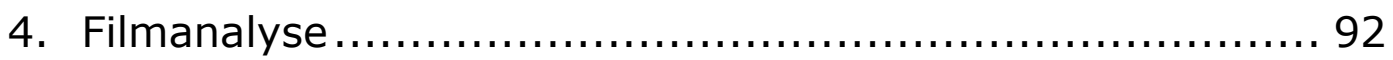

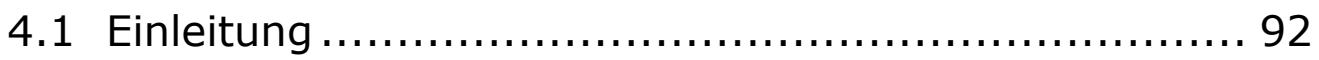

4.2 Sequenzprotokoll......................................... 94

4.3 Bildebene .............................................. 102

4.4 Bildgestaltung, Filmsprache ..........................107

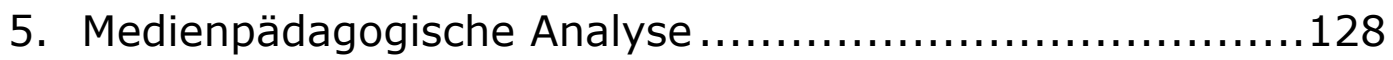

5.1 Emotionale Erlebnisqualität - Betroffenheit und Identifikation .......................................... 128

5.2 Die kognitive Erlebnisqualität .........................132

5.3 Die ästhetische Erlebnisqualität ........................133

5.4 Der Film als potentielles Lernfeld und als

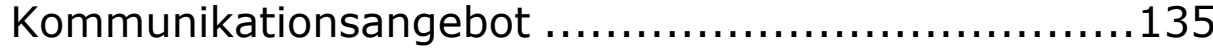

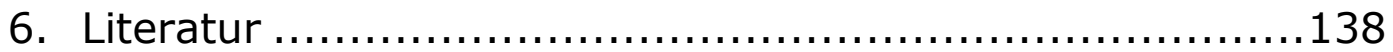




\section{Abbildungsverzeichnis}

Abbildung 1: Protagonist Jamal (re.) und sein Cousin Enayatullah. ................................................... 89

Abbildung 2: Jamal muss sich von nun an alleine durchschlagen............................................... 90

Abbildung 3: Die politische Karte gibt Aufschluss über den

Fortschritt der Reise. 102

Abbildung 4: Schriftzüge geben Aufschluss über die Orte des

Geschehens............................................... 103

Abbildung 5: Protagonist Jamal. ............................. 105

Abbildung 6: Jamals Cousin Enayatullah....................... 106

Abbildung 7: Dramenverlaufskurve für den Film „In This

World". .................................................... 107

Abbildung 8: Kinder im Shamshatoo. ......................... 112

Abbildung 9: Peshawar bei Nacht. ............................ 114

Abbildung 10: Gespräche mit den Schleusern. ................. 115

Abbildung 11: Die Karte fungiert immer wieder als roter

Faden der Fluchtroute. ..................................... 116

Abbildung 12: Freude und Gelassenheit trotz der

Fluchtumstände bei Jamal.

Abbildung 13: Enayatullah auf der Ladefläche eines Pick-Ups. 118 Abbildung 14: Jamal und Enayatullah bei einem Fluchthelfer. 119 Abbildung 15: Perspektive der Grenzer auf die Protagonisten. 120 Abbildung 16: Bei Nacht bezwingen die Protagonisten im

Schnee den Berg............................................ 121

Abbildung 17: Die letzten Momente von Enayatullah. .......... 122

Abbildung 18: Nach Ankunft in Triest rennt Jamal weg......... 123 Abbildung 19: Enayatullahs Vater hadert mit dem Schicksal seines Sohnes............................................... 125 


\section{Produktionsfakten}

\begin{tabular}{|c|c|}
\hline Deutscher Filmtitel & $\begin{array}{l}\text { In This World - Aufbruch ins } \\
\text { Ungewisse }\end{array}$ \\
\hline Regie & Michael Winterbottom \\
\hline Produktion & Revolution Films \\
\hline Verleih & Arsenal \\
\hline Buch & Toni Grisoni \\
\hline Kamera & Marcel Zyskind \\
\hline Schnitt & Peter Christelis \\
\hline Musik & Dario Marianelli \\
\hline Produktionsland & Großbritannien \\
\hline Erscheinungsjahr & 2002 \\
\hline Filmlänge & 85 Minuten \\
\hline Filmsprache & $\begin{array}{l}\text { Pashtu, Farsi, Englisch (mit dt. } \\
\text { Untertiteln) }\end{array}$ \\
\hline FSK & Ab 12 \\
\hline Preise & $\begin{array}{l}\text { Goldener Bär (Internationale } \\
\text { Filmfestspiele 2003) } \\
\text { Friedenspreis der Heinrich- } \\
\text { Böll-Stiftung (Berlinale 2003) } \\
\text { Preis der Ökumenischen Jury } \\
\text { (Berlinale 2003) }\end{array}$ \\
\hline
\end{tabular}




\section{Inhalt und Handlung}

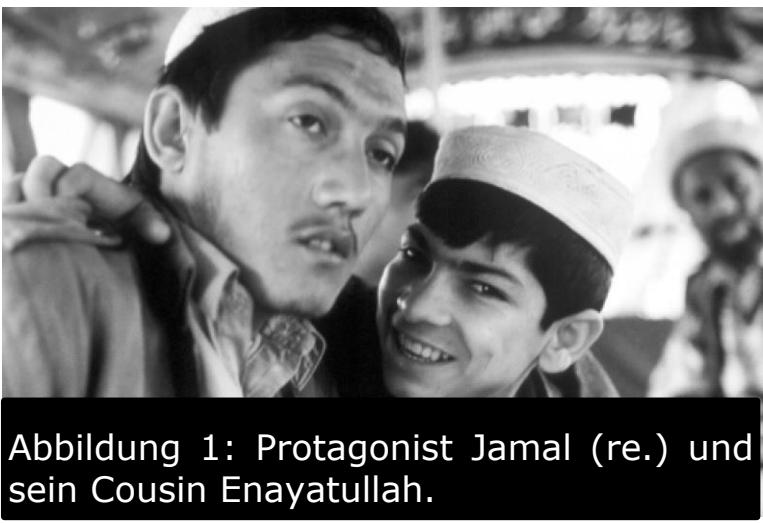

Februar 2002: Das Waisenkind Jamal arbeitet für nicht einmal einen Dollar pro Tag in einer Steinfabrik im pakistanischen Flüchtlingslager Shamshatoo. Sein Cousin Enayatullah, der in Peshawar am Marktstand seiner Familie arbeitet, träumt von einem besseren Leben in London. Weil Jamal allerdings der englischen Sprache mächtig ist, schickt ihn sein Onkel gemeinsam mit Enayatullah auf die lange und gefährliche Reise nach Großbritannien, wo sie Arbeit finden und viel Geld verdienen sollen.

Die Tickets dafür kauft er bei einem illegalen Schleuser, der die beiden in die Hände eines aus Menschenschmugglern bestehenden Netzwerkes gibt, das über die Kontinente hinweg gut ausgebaut zu sein scheint. Noch im selben Monat fahren Jamal und Enayatullah von Pakistan aus mit dem Bus durch das Land bis in den Iran, wo sie bei einer Grenzkontrolle aufgrund fehlender Ausweispapiere des Landes verwiesen werden.

In einer Wüste werden sie ausgesetzt und brauchen eine Woche, um zu Fuß und per Anhalter zurück nach Peshawar zu gelangen. Dort treffen sie sich mit dem Schmuggler, der sie zuvor in den Bus nach Teheran setzte. Doch den verpatzten Staatswechsel nimmt er nicht auf seine Kappe. Stattdessen fordert er für einen zweiten Versuch erneut Geld von den beiden Jungen.

Am 12. April erreichen sie nach einem weiteren Anlauf die iranische Hauptstadt. Einige Tage später fahren sie auf der Ladefläche eines Pick-Ups, versteckt zwischen Orangen, durch das Land bis zu einem kleinen Grenzort, wo sie von einer iranischen Familie herzlich aufgenommen und versorgt werden. Mit der Hilfe eines Jungen der Familie überqueren sie das verschneite Grenzgebirge, das das Land von der Türkei trennt. 
In der Dunkelheit der Nacht beobachten sie, wie Menschen von der Grenzpolizei erschossen werden. Doch Jamal und Enayatullah schaffen es zusammen mit einer flüchtenden Familie nach Istanbul. Hier arbeiten sie zunächst in einer Werkstatt, bis sie eines Tages von den engagierten Schmugglern abgeholt werden. Mit anderen Flüchtlingen und der Familie, die ein Kleinkind dabeihat, werden sie in einem luftdichten Container eingesperrt, der auf einen LKW geladen und hinter Paketen und Kisten versteckt wird. Als es den illegalen Passagieren zunehmend schlechter geht, rufen sie verzweifelt nach Hilfe, doch ihre Schreie bleiben ungehört.

Als Schmuggler den Container 40 Stunden später öffnen, haben nur Jamal und das Kleinkind überlebt, das sich weinend in die Arme seiner toten Mutter kuschelt. In der italienischen Hafenstadt Triest schlägt sich Jamal von nun an ohne Enayatullah und ohne

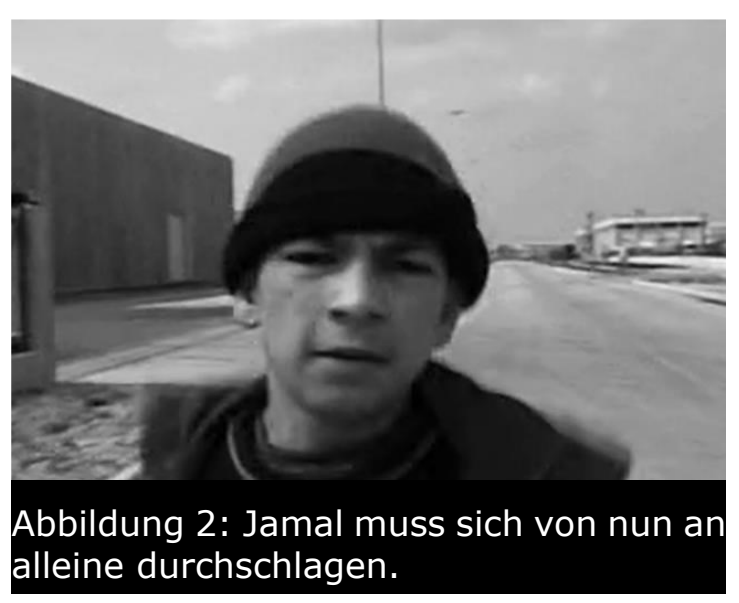
Geld durch das Leben. Durch das Verkaufen von Schmuck wird er nicht reich, also beklaut er die wohlhabenden Einwohner Italiens. Vom Geld kauft er sich ein Zugticket nach Paris und landet letztendlich in der französischen Hafenstadt Sangatte, von der aus regelmäßig Schiffe nach Großbritannien fahren.

In einem Flüchtlingslager trifft er auf einen jungen Mann, der bereits in London gelebt hat und immer noch die Schlüssel für das Restaurant, in dem er gearbeitet hat, besitzt. Eines nachts machen sie sich mit Holzbrettern ausgerüstet auf den Weg zu einer LKW-Parkfläche. Ihre Bretter klemmen sie unter einen geeigneten Lastwagen, der durch den Eurotunnel von Frankreich aus nach Großbritannien fährt.

Auf den Brettern liegend verharren sie unter den schweren Lastern, bis diese das Festland erreichen. Im Juni 2002 ist Jamal nach vier Monaten in London angekommen, wo er ebenfalls in einem Restaurant Arbeit gefunden hat. In einem Telefonat mit seinem Onkel muss er schmerzlichst gestehen, dass Enayatullah auf der Reise gestorben ist. Jamal stellt in London einen Antrag auf Asyl, 
welcher kurz darauf abgelehnt wird. Allerdings erhält er eine Ausnahmegenehmigung für ein Bleiberecht bis einen Tag vor seinen 18. Geburtstag. Dann muss er das Land verlassen haben.

\section{Sozialhistorische Analyse}

Der Film behandelt die Thematiken der immer noch andauernden Kriege zwischen Afghanistan und Pakistan. Shamshatoo, das Flüchtlingslager indem der Protagonist Jamal lebt, liegt 30 Kilometer westlich von Peshawar (vgl. Ladurner 2001). Dieses Flüchtlingslager wurde in den 1980er Jahren wegen des Sowjetisch-Afghanischen Krieges errichtet. Die sowjetischen Interventionen in Afghanistan begannen im Dezember 1979. Die Konfliktparteien waren Afghanistan und die Mudschahedin. Der Grund für diese Auseinandersetzung war das militärische Eingreifen seitens der Regierung gegen die zahlreichen Gruppierungen afghanischer Mudschahedin.

Eine Ausbreitung des radikalen Islams auf zentralasiatische Sowjetrepubliken sollte verhindert werden. Allerdings scheiterten die Interventionen, sodass die sowjetischen Truppen abzogen und die gegnerischen Konfliktparteien die Macht übernahmen. Durch das Ende der sowjetischen Regierungen und die gescheiterten Verständigungsversuche zwischen den rivalisierenden Mudschahedinparteien begann 1989 der Bürgerkrieg in Afghanistan (vgl. Ruttig 2014). Folglich stieg der Islamismus auf und somit auch die Herrschaft der Talibanorganisation. Die Taliban eroberte die Hauptstadt und nahmen im gleichen Atemzug Osama bin Laden und seine Al-Qaida Organisation auf.

Nach dieser Machtübernahme besaßen die Taliban $63 \%$ des Landes (vgl. Khalatbari 2008). Die weiteren Prozente teilten sich zwei weitere Kräftezentren. Ein Krieg zwischen den Taliban und den Truppen Massouds sollten zur Übernahme des ganzen Landes führen. Nach Beendigung dessen war das Land praktisch entvölkert und die Infrastruktur völlig zerstört.

Da die Truppen Massouds nicht besiegt wurde, wurde am 09. September 2001 der Anführer ermordet. Zwei Tage später erfolgten die terroristischen Anschläge in den USA. Die ausführenden Täter 
wurden als Mitglieder der Al-Qaida identifiziert. Daraufhin forderte der UN-Sicherheitsrats eine sofortige und bedingungslose Auslieferung Osama bin Ladens (vgl. Kellerhoff 2010). Die Taliban verweigerten diesen Befehl allerdings, woraufhin ihr Regime in Afghanistan gestürzt wurde. Die Talibanführung floh nach Pakistan. Sie hinterließen eine völlig zerstörte Zivilbevölkerung, misshandelte Menschen und zahlreiche Todesopfer.

\section{Problemstellung}

Winterbottom möchte genau das widerspiegeln. Er stellte den Zusammenhang zwischen den Kosten für den Krieg gegen die Taliban und der Tatsache her, dass fast niemand gewillt ist, für die damit ruinierten unschuldigen Leben Geld aufzubringen oder auch nur ein bisschen Verantwortung zu übernehmen. Er unterstreicht sein Anliegen, auf die unsinnigen Unterscheidungen zwischen anscheinend würdigen und unwürdigen Menschen, zwischen politisch Verfolgten und sogenannten Wirtschaftsflüchtlingen hinzuweisen.

\section{Filmanalyse}

\subsection{Einleitung}

"In This World" - Aufbruch ins Ungewisse ist ein 2002 erschienener Spielfilm von Michael Winterbottom. Das dazugehörige Drehbuch von Tony Grisoni bestand vor Drehbeginn lediglich aus einem oberflächlichen Konstrukt, das bestimmte Ideen und einen eher allgemeinen Handlungsfaden beinhaltete. Vielmehr entstand der Film aus einer Spontanität heraus, die wesentlich zur Authentizität beiträgt.

Eben dieser Spontanität entsprang letztendlich eine Semi-Dokumentation, die die Thematik der Flüchtlingspolitik und deren Konsequenzen aufzuzeigen versucht, indem Laiendarsteller vor Ort gecastet und in den Dreh eingebunden wurden, ohne, dass konkrete Handlungsabläufe vorab festgelegt wurden. Und so folgten die Filmemacher gemeinsam mit den beiden Hauptdarstellern einer ausgebauten Flüchtlingsroute von Pakistan aus über den Iran, die Türkei, Italien und Frankreich bis hin nach Großbritannien, wo 
sie viele weitere Flüchtende trafen, die von ihren Reisen berichteten.

All diese Erfahrungen und Gespräche konnten in den Filmdreh eingebunden werden, so dass das letztendliche Konzept aus den einzelnen Etappen und ihren kulturellpolitischen Einflüssen schrittweise perfektioniert wurde. Auf diese Weise wirkt "In This World" wie die Dokumentation tatsächlicher Erlebnisse, die uns Zuschauer für die Thematik sensibilisiert.

Denn obwohl wir mit den Problemen und den Konsequenzen solcher oder ähnlicher Bewegungen vertraut sind, ist das damit einhergehende Leid und die Gefahr von außen nicht eindeutig nachvollziehbar. An dieser Stelle setzt der Film an und macht sichtbar, was Flüchtende auf ihren langen Reisen erleben. So wird der Zuschauer unmittelbar selbst zum Betroffenen und erfährt, was es heißt, sich in solch eine bedrohliche Situation zu begeben. 


\subsection{Sequenzprotokoll}

\begin{tabular}{|l|l|}
\hline Sequenz & Inhalt \\
\hline Sequenz 1 & Titelvorspann mit orientalischer Musik \\
\hline Sequenz 2 & $\begin{array}{l}\text { Bilder vom Flüchtlingslager "Shamshatoo" im Nordwesten Pakistans werden gezeigt. Es ist } \\
\text { Februar 2002. Verschiedene Szenen vom Ort und Menschen, insbesondere Kindern, werden } \\
\text { bei alltäglichen Tätigkeiten gefilmt. Ein Sprecher (Voice-Over) berichtet: "53000 afghani- } \\
\text { sche Flüchtlinge leben im Lager bei Peshawar in Pakistan. Die ersten trafen 1979 ein nach } \\
\text { der sowjetischen Invasion ihres Landes. Die letzten nach der Bombardierung, die am 7. } \\
\text { Oktober 2001 begann." Erster Hauptcharakter wird vorgestellt: "Vieler dieser Kinder sind } \\
\text { hier geboren, wie Jamal. Er ist Waise, arbeitet in einer Steinfabrik und verdient weniger als } \\
\text { einen Dollar am Tag." Voice-Over: "2001 wurden 7,9 Milliarden Dollar für die Bombardie- } \\
\text { rung Afghanistans ausgegeben. Bei Flüchtlingen ist man nicht großügig: Eine tägliche Es- } \\
\text { sensration sind 480 Gramm Weizenmehl, 25 Gramm Pflanzenöl und 60 Gramm Hülsen- } \\
\text { früchte. Jede Familie erhält ein Zelt, eine Plastikplane, drei Decken und einen Ofen." Jamal } \\
\text { sucht seine Kleidung. Dann macht er sich auf den Weg in die Stadt zu seinem Onkel }\end{array}$ \\
\hline Sequenz 3 & $\begin{array}{l}\text { Jamal gelangt mit einem Laster, dann auch mit einem Bus in die Stadt, wo er auf eine } \\
\text { Menschenmasse zugeht. Zwischen den vielen Männern steht sein Onkel. Sie begrüßen sich } \\
\text { herzlich und essen gemeinsam. Zusammen mit Jamals Cousin Enayatullah beschließt die } \\
\text { Gruppe, dass die beiden nach London gehen sollen. Dann wird weiter getanzt und gefeiert. }\end{array}$ \\
\hline
\end{tabular}




\begin{tabular}{|c|c|}
\hline Sequenz 4 & $\begin{array}{l}\text { Die Männer suchen einen Schleuser auf. Der Schleuser macht der Familie einen Preisvor- } \\
\text { schlag. }\end{array}$ \\
\hline Sequenz 5 & mal erhält einen Anruf von seinem Onkel und fährt erneut nach Peshawar. \\
\hline z 6 & $\begin{array}{l}\text { Auf dem Basar ist viel los. Erneut führen die Männer Gespräche mit den Schleusern. Jamal } \\
\text { und Enayatullah sollen über den Landweg nach London gelangen. Das sei eine gefährliche } \\
\text { Strecke. Der Schleuser zählt das Bündel Geld, das ihm Enayatullah's Vater gegeben hat. } \\
\text { Der Schleuser selbst verspricht, dass er das Geld erst bekommt, wenn die beiden Cousins } \\
\text { in London angekommen sind und ihren Onkel anrufen. Sie bekommen eine Quittung, die } \\
\text { sie aufheben müssen. In ausgelassener Runde wird gefeiert. Sie bekommen Geschenke zum } \\
\text { Abschied. }\end{array}$ \\
\hline Sequenz 7 & Jamal und Enayatullah nehmen Abschied von ihren Familien und Freunden. \\
\hline Seq & $\begin{array}{l}\text { Jamal und Enayatullah kommen am Treffpunkt an. Sie bekommen Ausweise, Telefonnum- } \\
\text { mern und Fahrkarten zugesteckt. Der Bus fährt los und die beiden winken ihren Familien } \\
\text { zu. Gesenkte Blicke und Emotionen auf beiden Seiten sind zu spüren. }\end{array}$ \\
\hline Sequenz 9 & $\begin{array}{l}\text { Eine animierte Weltkugel wird eingeblendet. Wieder spricht das Voice-Over: „Es gibt } 14,5 \\
\text { Millionen Flüchtlinge auf der Welt. Mehr als } 5 \text { Millionen leben in Asien. Davon lebt fast } 1 \\
\text { Millionen in Peshawar. Viele von ihnen wollen gehen." Es wird auf eine politische Karte } \\
\text { gezoomt, die den pakistanischen Staat zeigt. Mit Hilfe einer roten Linie wird der Weg ge- } \\
\text { kennzeichnet, den Jamal und Enayatullah während ihrer Reise zurücklegen. Die rote Linie } \\
\text { stoppt bei Quetta. Der Sprecher berichtet weiter: "Jährlich begeben sich } 1 \text { Millionen Men- } \\
\text { schen in die Hände von Menschenschmugglern. Einigen gelingt die Flucht, viele werden von }\end{array}$ \\
\hline
\end{tabular}




\begin{tabular}{|l|l|}
\hline & $\begin{array}{l}\text { den Behörden aufgegriffen. Und einige sterben auf ihrem Weg." Szenen folgen, in denen } \\
\text { Kinder selbstgebaute Papierdrachen steigen lassen. Es werden Impressionen von Quetta } \\
\text { gezeigt. }\end{array}$ \\
\hline Sequenz 10 & $\begin{array}{l}\text { Vom Busbahnhof aus fahren Jamal und Enayatullah mit einem Taxi zur Unterkunft, wo sie } \\
\text { übernachten werden. Abends im Bett sprechen sie über die Entstehung der Musik und er- } \\
\text { zählen sich erfundene Geschichten, über die sie herzlich lachen. }\end{array}$ \\
\hline Sequenz 11 & $\begin{array}{l}\text { Am nächsten Tag genießen die beiden Cousins den Ausblick auf die Stadt. Durch das Ge- } \\
\text { wimmel der Straßen schaffen sie es nicht rechtzeitig, am nächsten Treffpunkt anzukommen. } \\
\text { Der Fahrer ist sauer. Es folgen nun mehrere Fahrzeugwechsel. Die Jungen steigen mehrfach } \\
\text { um, fahren auf Pick-Ups, in LKW's oder Bussen, zwischendurch waschen sie sich, essen und } \\
\text { beten. In einer Kneipe treffen sie sich mit dem nächsten Flüchtlingshelfer. }\end{array}$ \\
\hline Sequenz 12 & $\begin{array}{l}\text { Das Auto, in dem sie mitfahren, wird angehalten und kontrolliert. Jamal und Enayatullah } \\
\text { geben an, sie seien beruflich unterwegs und würden Kleidung für ihren Laden kaufen wollen. } \\
\text { Die Beamten (wahrscheinlich Soldaten) kontrollieren ihr Gepäck. Um ihn zu beschwichtigen, } \\
\text { bietet Jamal dem Soldaten Eneyatullah's Walkman an. Anschließend dürfen sie weiterreisen, } \\
\text { doch Enayatullah ist sauer darüber, dass Jamal ohne Einverständnis seinen Walkman ver- } \\
\text { schenkt hat. }\end{array}$ \\
\hline Sequenz 13 & $\begin{array}{l}\text { Erneut wird die politische Karte gezeigt. Von Quetta aus zieht sich der rote Faden nach } \\
\text { Taftan. Hier kommen sie in einem Hotel an, in dem sie mit einem Mann verabredet sind. } \\
\text { Bei ihm tauschen sie Geld zu angeblich guten Konditionen um, was als Notwendigkeit für }\end{array}$ \\
\hline
\end{tabular}




\begin{tabular}{|l|l|}
\hline & $\begin{array}{l}\text { den ersten Staatswechsel in den Iran gesehen wird. Dann fahren sie mit dem Mann mit. } \\
\text { Der Mann übergibt sie an einen Fahrer in einem Pickup, der sie über die Grenze schleust. }\end{array}$ \\
\hline Sequenz 14 & $\begin{array}{l}\text { Der Staatswechsel scheint zunächst erfolgreich. Im Iran treffen sie einen weiteren Flücht- } \\
\text { lingshelfer, der ihnen neue Kleidung gibt, um möglichst unauffällig zu sein. Auch ihre Kap- } \\
\text { pen müssen sie abgeben. Der Mann spricht nur Englisch und kann deshalb nur mit Jamal } \\
\text { kommunizieren. Enayatullah muss immer wieder nachfragen, und wirft misstrauische Be- } \\
\text { merkungen in den Raum. Der Mann selbst will auch wissen, was Enayatullah sagt. Der Mann } \\
\text { vermietet für viel Geld ein Zimmer an die beiden. Die beiden Jungs fühlen sich betrogen. } \\
\text { Sie müssen zehn Tage dort verharren, bis sie weiterreisen können. }\end{array}$ \\
\hline Sequenz 15 & $\begin{array}{l}\text { Mit dem Mann fahren die beiden zum Busbahnhof, wo sie in einen Bus einsteigen. Von dem } \\
\text { Mann bekommen sie anschließend die nächste Kontaktnummer vom Helfer in Teheran, was } \\
\text { das nächste Ziel ist. Doch nach kurzer Fahrt wird der volle Bus angehalten. Es erfolgt eine } \\
\text { Kontrolle. Jamal und Enayatullah können sich nicht ausweisen. Man hält sie für Afghanen, } \\
\text { denn obwohl sie sich als Iraner ausgeben, können sie das nicht mit Papieren belegen. Sie } \\
\text { werden des Busses verwiesen. Man kontrolliert ihr Gepäck. Dann setzt man sie auf die } \\
\text { Ladefläche eines Pickups, auf welchem sich auch ein Maschinengewähr befindet. In einer } \\
\text { Wüste setzt man sie aus. }\end{array}$ \\
\hline Sequenz 16 & $\begin{array}{l}\text { Jamal und Enayatullah laufen durch die Wüste. Nach einer Woche sind sie zurück in Pesha- } \\
\text { war, Pakistan. Der Versuch, nach Teheran zu gelangen, ist gescheitert. }\end{array}$ \\
\hline Sequenz 17 & $\begin{array}{l}\text { Die beiden Cousins sitzen wieder beim Schleuser, bei dem sie zuvor ihr Geld umgetauscht } \\
\text { hatten. Der wiederum fühlt sich nicht verantwortlich für die Jungen und fordert für den }\end{array}$ \\
\hline
\end{tabular}




\begin{tabular}{|l|l|}
\hline & $\begin{array}{l}\text { zweiten Versuch erneut Geld. Enayatullah holt daraufhin Geld aus seinem Schuh, das er } \\
\text { zuvor dort versteckt hat. }\end{array}$ \\
\hline Sequenz 18 & $\begin{array}{l}\text { Der zweite Versuch ist erfolgreich. Jamal und Enayatullah erreichen mit einem Bus Teheran, } \\
\text { die iranische Hauptstadt. Es folgen Bilder der iranischen Großstadt, die zudem viel moderner } \\
\text { und hektischer wirkt, als Peshawar. }\end{array}$ \\
\hline Sequenz 19 & $\begin{array}{l}\text { Es ist der 22. April 2002. Jamal und Enayatullah können ihren Kontaktmann nicht erreichen. } \\
\text { Sie schlendern durch die Straßen Teherans, essen Eis und schauen in ihrem Hotelzimmer } \\
\text { TV. }\end{array}$ \\
\hline Sequenz 20 & $\begin{array}{l}\text { 5 Tage später fahren Jamal und Enayatullah in einem blauen Auto in ein Lager. Ein Mann } \\
\text { schreit innen zu, sie sollen sich beeilen. Enayatullah wehrt sich zunächst, setzt sich dann } \\
\text { aber doch gemeinsam mit Jamal auf die Ladefläche eines Lasters. Männer stapeln mehrere } \\
\text { Kisten voller Orangen um die beiden Jungen herum. }\end{array}$ \\
\hline Sequenz 21 & $\begin{array}{l}\text { Erneut wechseln sie das Fahrzeug. Der Fahrer spricht eine andere Sprache, die den beiden } \\
\text { nicht verständlich ist. In einem kleinen Dorf nahe der türkischen Grenze werden sie von } \\
\text { einer iranischen Familie herzlich aufgenommen. Sie bekommen Essen und Trinken, werden } \\
\text { mit neuen Schuhen beschenkt. Man segnet und betet für die beiden, die in den Augen der } \\
\text { Frauen noch Kinder sind, für die man Mitleid empfindet. Am Abend wird gefeiert und aus- } \\
\text { gelassen getanzt. }\end{array}$ \\
\hline Sequenz 22 & $\begin{array}{l}\text { Mit einem kleinen Jungen der Familie machen sich Jamal und Enayatullah auf den Weg zur } \\
\text { Grenze. Dabei müssen sie die verschneiten Grenzgebirge überqueren. Der Schnee ist so } \\
\text { tief, dass sie kaum laufen können. Es schneit und es ist kalt. Um möglichst unerkannt zu }\end{array}$ \\
\hline
\end{tabular}




\begin{tabular}{|l|l|}
\hline & $\begin{array}{l}\text { bleiben, wandern sie in der Nacht durch das verfrorene Gebirge. Schweratmend und völlig } \\
\text { erschöpft hören sie Schüsse. Die Grenzbeamte schießen auf illegale Grenzgänger. Der } \\
\text { kleine Sohn der Familie will umkehren. Leise verstecken sie sich im Schnee und ziehen dann } \\
\text { schließlich weiter. }\end{array}$ \\
\hline Sequenz 23 & $\begin{array}{l}\text { In der Türkei angekommen fahren die beiden Jungen auf einer Ladefläche eines LKW's wei- } \\
\text { ter. Dort treffen sie auf eine Familie bestehend aus Vater, Mutter und einem Kleinkind. } \\
\text { Diese möchten nach Dänemark flüchten. }\end{array}$ \\
\hline Sequenz 24 & $\begin{array}{l}\text { Jamal, Enayatullah und die Familie kommen in Istanbul an. Sie treffen sich in einem Res- } \\
\text { taurant mit dem nächsten Flüchtlingshelfer, der sie anschließend zur Unterkunft bringt, wo } \\
\text { auch andere illegale Flüchtlinge leben. }\end{array}$ \\
\hline Sequenz 25 & $\begin{array}{l}\text { Am nächsten Tag werden Jamal und Enayatullah von den anderen illegalen Bewohnern des } \\
\text { Hauses zu einer Werkstatt geführt, in welcher sie arbeiten sollen. In den nächsten Tagen } \\
\text { schauen sie sich die türkische Hauptstadt an, gehen essen, verbringen Zeit mit der Familie } \\
\text { und arbeiten täglich. }\end{array}$ \\
\hline Sequenz 26 & $\begin{array}{l}\text { Nach einiger Zeit werden die beiden Jungen in der Werkstatt von den nächsten Menschen- } \\
\text { schmugglern abgeholt. Gemeinsam mit der Familie und weiteren Flüchtlingen werden sie in } \\
\text { einem LKW untergebracht, der sie zum Hafen fährt. Um auf dem Schiff nicht aufzufallen, } \\
\text { schließt man sie in einem luftundurchlässigen Container ein, welcher sich auf der Ladefläche } \\
\text { eines LKW's hinter einer Fracht befindet. }\end{array}$ \\
\hline Sequenz 27 \\
$\begin{array}{l}\text { Die Schifffahrt beginnt und die politische Karte zeigt den rot markierten Weg über das Meer } \\
\text { von Istanbul nach Italien. Währenddessen merken die Flüchtlinge im Container, wie es }\end{array}$ \\
\hline
\end{tabular}




\begin{tabular}{|l|l|}
\hline & $\begin{array}{l}\text { ihnen zunehmend schlechter geht. Das Baby schreit ununterbrochen. Sie haben nur eine } \\
\text { Taschenlampe, die kleine Lichtpunkte im Raum ermöglicht. Langsam wird die Luft knapp } \\
\text { und die Menschen schreien panisch, klopfen dabei an die Türen. Keiner hört sie. Plötzlich } \\
\text { wird Enayatullah ohnmächtig. }\end{array}$ \\
\hline Sequenz 28 & $\begin{array}{l}40 \text { Stunden später hat das Schiff die italienische Hafenstadt Trieste erreicht. Als die dort } \\
\text { anwesenden Flüchtlingshelfer den Container öffnen, kommt Jamal nur langsam zu Bewusst- } \\
\text { sein. Auch das Baby beginnt nach Luft zu schnappen und schreit in den Armen seiner schon } \\
\text { toten Mutter. Auch Enayatullah und die anderen Flüchtlinge haben die Überfahrt nach Italien } \\
\text { nicht übererlebt. Als Jamal begreift, was gerade geschehen ist, fängt er an zu rennen. }\end{array}$ \\
\hline Sequenz 29 & $\begin{array}{l}\text { 2 Wochen später läuft Jamal alleine und ohne Geld durch die Straßen Italiens, wo er einfa- } \\
\text { che Straßenware verkauft. Um an mehr Geld zu gelangen, beklaut er eine wohlhabende } \\
\text { Familie, die ihm zuvor etwas Kleingeld zugesteckt hat. }\end{array}$ \\
\hline Sequenz 30 & $\begin{array}{l}\text { Von dem Geld, das Jamal aus der Handtasche der Frau geklaut hat, kauft er sich am Bahn- } \\
\text { hof ein Ticket nach Paris. Mit dem Zug fährt er nach Frankreich. Die politische Karte kenn- } \\
\text { zeichnet den Weg von Trieste aus mit einer roten Linie. Sie endet in Sangatte, wo sich ein } \\
\text { Auffanglager für Flüchtlinge befindet. }\end{array}$ \\
\hline Sequenz 31 & $\begin{array}{l}\text { Im Lager teilt er sich ein Zelt mit einem jungen Mann, der bereits in London gearbeitet hat. } \\
\text { Er besitzt immer noch die Schlüssel vom Restaurant, in welchem er tätig war, und möchte } \\
\text { die Überfahrt erneut riskieren. Die beiden freunden sich an. }\end{array}$ \\
\hline Sequenz 32 & $\begin{array}{l}\text { Am 6. Juni 2002 starten die beiden Freunde in der Abenddämmerung vom Lager aus los. } \\
\text { Sie haben zwei Holzbretter dabei, die sie unter einen LKW klemmen. Dann legen sie sich }\end{array}$ \\
\hline
\end{tabular}




\begin{tabular}{|l|l|}
\hline & $\begin{array}{l}\text { auf die Bretter. Der LKW wird auf einen Güterzug geladen, welcher durch den Kanaltunnel } \\
\text { nach Großbritannien fährt. }\end{array}$ \\
\hline Sequenz 33 & $\begin{array}{l}\text { In London angekommen arbeitet auch Jamal als Aushilfskraft in einem Restaurant. Von dort } \\
\text { bekommt er die Gelegenheit, bei seinem Onkel anzurufen. Er erzählt ihm, dass er endlich } \\
\text { in London gelandet ist. Der Onkel fragt nach Enayatullah. Jamal muss ihm gestehen, dass } \\
\text { er "nicht mehr auf dieser Welt lebt”. Jamals Onkel senkt den Kopf und verliert die Sprache. }\end{array}$ \\
\hline Sequenz 34 & $\begin{array}{l}\text { Es folgen Bilder aus Shamshatoo. Kinder lachen, alles ist wie gewohnt. Die Menschen leben } \\
\text { weiter, und auch Jamals Onkel lebt in trauriger Gewissheit sein Leben weiter. Währenddes- } \\
\text { sen besucht Jamal in London eine Moschee, in welcher er betet und seine Trauer Kund gibt. } \\
\text { Die Strapazen der Reise und die schrecklichen Erlebnisse haben ihm sichtbar zugesetzt. Er } \\
\text { weint }\end{array}$ \\
\hline Sequenz 35 & $\begin{array}{l}\text { Der Sprecher beendet den Film mit folgenden Informationen: "Am 9.8.2002 wurde Jamal } \\
\text { Udin Torabi's Asylantrag abgelehnt. Er durfte jedoch mit Ausnahmegenehmigung einreisen } \\
\text { und lebt jetzt in London. Er muss das Land einen Tag vor seinem 18. Geburtstag verlassen." }\end{array}$ \\
\hline Sequenz 36 & \begin{tabular}{l} 
Es folgt der Abspann, im Hintergrund orientalische Musik und das flüsternde Beten Jamals. \\
\hline
\end{tabular}
\end{tabular}




\subsection{Bildebene}

\section{Orte, Umgebung und Ausstattung}

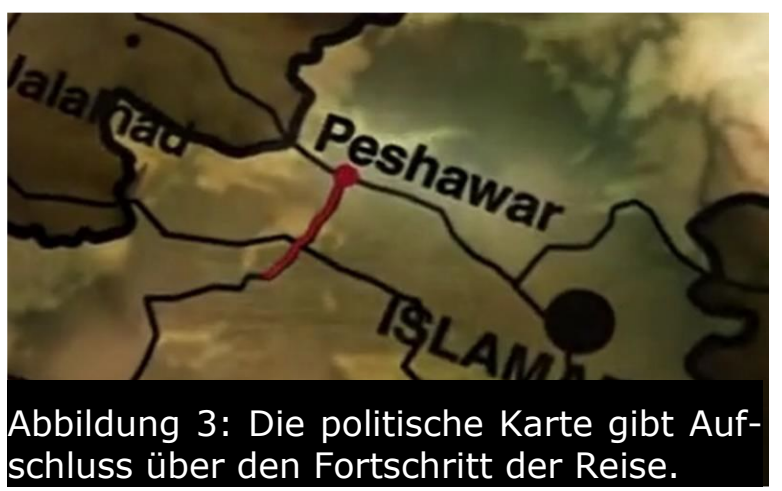

Gezeigte Orte und Umgebungen entsprechen den im Film angegebenen und auf der politischen Karte markierten Drehorten. Eine eingeblendete, animierte Karte zeigt die Staaten, deren Grenzen, die Hauptstädte, sowie die Orte, an denen Jamal und Enayatullah Halt machen bzw. welche sie auf ihre Reise durchqueren. So kann der Zuschauer den langen und beschwerlichen Weg der beiden Jungen nachvollziehen und sie auf ihrer Reise begleiten.

Start ist die pakistanische Metropole Peshawar im Nordwesten des Landes. Von dort reisen die Jungen zunächst nach Quetta, bevor sie iranisches Territorium betreten. Als der Versuch, nach Teheran zu gelangen, scheitert, landen Jamal und Enayatullah wieder in Peshawar, wo die Reise von Vorne beginnt.

Beim zweiten Anlauf schaffen sie es schließlich nach Teheran und damit in die iranische Hauptstadt. Nach wenigen Tagen Aufenthalt fahren sie zu einer kleinen Ortschaft, die sich an der Grenze zur Türkei befindet. Von hier aus müssen sie das verschneite Grenzgebirge überqueren, um nach Istanbul zu gelangen.

Auch in Istanbul verbringen sie ein paar Tage und arbeiten dort zusammen mit anderen illegalen Flüchtlingen in einer Werkstatt. Menschenschmuggler bringen sie später in einem Container unter, der sich auf der Ladefläche eines LKW's an Board eines Schiffes befindet. Die Reise geht nun über das Meer, vorbei an Griechenland bis nach Italien.

In der italienischen Hafenstadt Trieste muss sich Jamal von nun an selbst durchschlagen. Durch das Stehlen von Geld kann er sich schließlich ein Ticket nach Paris leisten, landet dann aber im französischen Sangatte, an der nördlichen Küste Frankreichs. Auch 
hier gibt es ein Auffanglanger für Flüchtlinge. Durch den Kanaltunnel gelangt Jamal mit seinem neuen Freund nach Großbritannien. In London findet er schließlich Arbeit.

Alle hier genannten Orte entsprechen den einzelnen Drehorten des Filmes. Die jeweiligen Etappen und Zwischenhalte sowie die dortigen Erlebnisse der beiden Jungen werden dokumentarisch festgehalten und begleitet.

An jeder neuen Etappe werden zunächst Impressionen und Bilder der Orte und der Menschen eingeblendet, die ihren Alltag in diesen auf unterschiedliche Weise meistern. So werden die Umgebungen dem Zuschauer vorgestellt, bevor sich die Handlungen in den jeweiligen Orten abspielen.

Des Weiteren erhält man einen Eindruck über die zum Großteil ärmlichen Verhältnisse der Menschen in den asiatischen Ländern, die in einem starken Kontrast zu den wohlhabenden Lebensweisen der Europäer stehen, was insbesondere in der italienischen Hafenstadt Trieste gut beobachtet werden kann.

Innerhalb der Orte erfolgt ein oft schneller Szenenwechsel, in welchem Landschaften, Straßen, Gassen, Häuser oder Plätze vorgestellt werden. Besonders rasant werden die Szenen in Großstädten aneinandergereiht, so dass die dort herrschende Hektik, insbesondere auf den Basaren oder Märkten, gut zum Ausdruck gebracht wird.

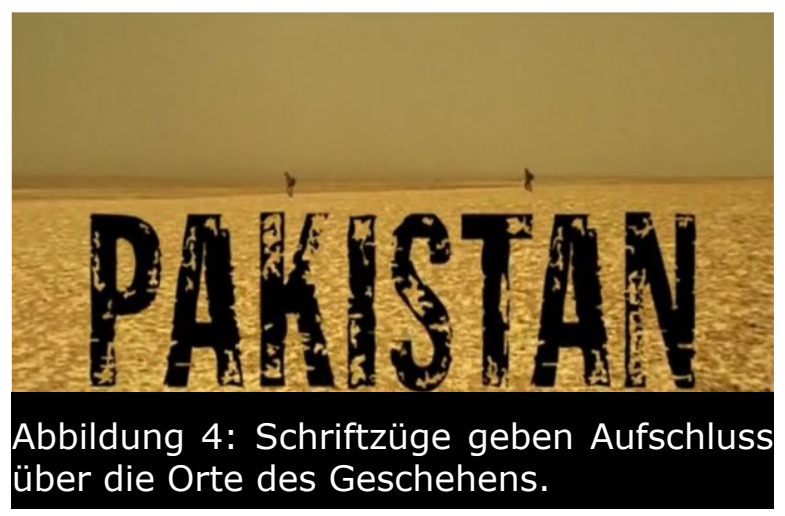

Jede neue Etappe wird zudem auf einer Szene, die den Ausblick auf den Ort oder die Straße bzw. den Weg zum Ort zeigt, schriftlich gekennzeichnet. So weiß der Zuschauer immer genau, wo sich Jamal und Enayatullah gerade befinden.

Zwischen den Orten werden zudem Szenen eingeblendet, die die beiden Jungen auf ihren Wegen begleiten. Gezeigt werden Hauptstraßen zwischen Wüstenlandschaften und holprige Wege auf inoffiziellen Fahrrouten, teilweise auch weitreichende Wüsten ohne 
weitere Anhaltspunkte. Auch mit tiefem Schnee bedeckte Grenzgebirge sind Bestandteil der Fluchtstrecke. Sowohl Schauspieler als auch am Film Beteiligte unternahmen die selbe Reise zu den angegebenen Orten, was den Film wie eine Dokumentation und gerade deshalb so authentisch wirken lässt.

Viele Szenen des Filmes entstanden spontan mit Hilfe der in den Orten lebenden Menschen oder basieren auf Gesprächen, die die Produzenten mit Flüchtlingen geführt haben. Somit wurden keine bestimmten Requisiten mitgebracht. Vielmehr wurde auf ganz alltägliche Handlungen oder typische Situationen in den jeweiligen Orten gesetzt, so dass die Szenen spontan aus den Laiendarstellen in den jeweiligen Orten und ihren Möglichkeiten zur Teilnahme entstanden.

Die einzige Ausnahme ist das Flüchtlingslager in Sangatte an der nordfranzösischen Grenze: Hier erhielt man keine Drehgenehmigung für den Film, weshalb das Lager nachgebaut werden musste. Diese Information bleibt beim Zuschauer allerdings unbemerkt, so dass der Film nicht an Authentizität verliert.

\section{Darsteller}

Der Film wird ausschließlich von Laiendarstellern gespielt. Die Nebenrollen wurden spontan in den jeweiligen Orten besetzt. Enayatullah wurde vom Regisseur auf einem Markt in Peshawar angesprochen, wo er mit gebrochenem Englisch versuchte, Waren an den Mann zu bringen. Jamal hingegen wurde nicht im Flüchtlingslager in Shamshatoo entdeckt, sondern in einer Sprachschule in Peshawar gecastet.

Die englische Sprache, der Jamal mächtig ist, war bedeutsam sowohl für die Kommunikation mit den Filmemachern als auch mit einzelnen Fluchthelfern während des gesamten Drehs. Dennoch entsprechen die Hintergründe der beiden jungen Männer zum größten Teil der Realität und im Film spielen nahezu alle Beteiligten sich selbst.

Bis auf Jamal und Enayatullah übernahmen die Laiendarsteller unverbindliche kleinere Rollen in den jeweiligen Orten, in denen sie lebten oder sich derzeit befanden, teilweise bei ihren alltäglichen 
Arbeiten. Nur Jamal und Enayatullah selbst wurden von den Filmemachern längerfristig und für den gesamten Filmdreh arrangiert.

So machten sie sich gemeinsam mit den Filmproduzenten auf den Weg von Pakistan nach London und stellten dabei eine realistische Fluchtroute von Flüchtlingen aus den betroffenen Gebieten dar. Die Beteiligten durchliefen die Fluchtstationen also selbst, wodurch eine gewisse dokumentarische Authentizität zustande kommt.

Für den Einsatz und die Filmteilnahme wurden die Hauptdarsteller mit Geld entlohnt. Ihnen wurden keine Versprechungen auf ein dauerhaft gültiges Visum im Ankunftsland gemacht.

Dennoch unternahm Jamal mit Hilfe seines noch gültigen Visums nach Abschluss der Dreharbeiten eine erneute Reise von Pakistan nach London, wo er einen Antrag auf Asyl stellte. Dieser wurde abgelehnt, jedoch erhielt er ein Bleiberecht bis zu einen Tag vor seinem 18. Geburtstag. Enayatullah hingehen baute sich in Peshawar von dem Geld eine Selbstständigkeit auf.

\section{Die Hauptcharaktere}

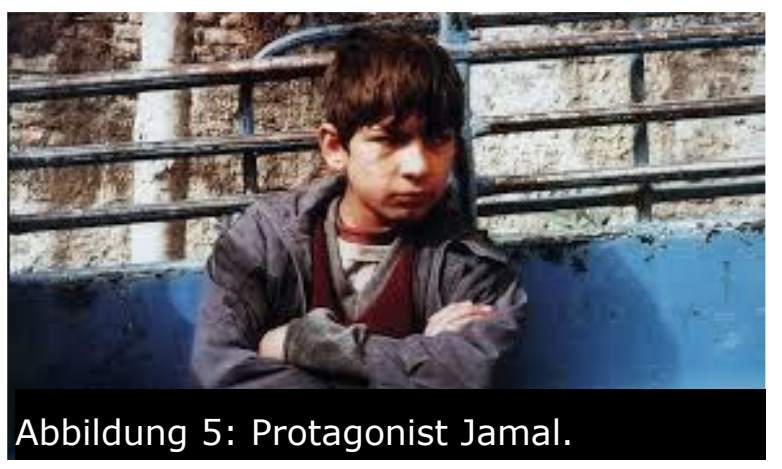

Jamal lebt als Waise im Flüchtlingslager in Shamshatoo und arbeitet dort in einer Steinfabrik für weniger als einen Dollar pro Tag. Er ist, wie die meisten Menschen im Lager, ein afghanischer Flüchtling. Zum familiären Hintergrund oder warum er Waise ist, wird im Film nichts gesagt. Gemeinsam mit anderen Kindern und Männern wohnt er in einem kleinen Haus. Er spricht fließend Englisch und wirkt sehr selbstständig und erwachsen. 


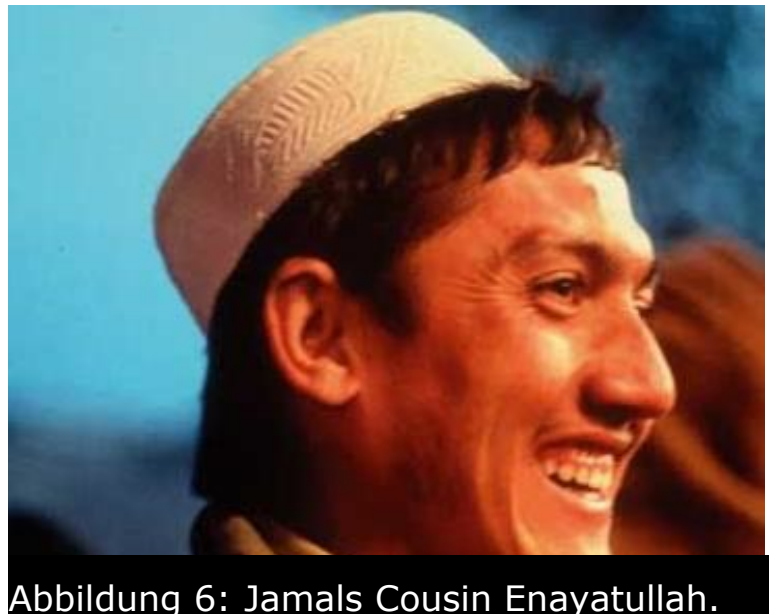

In Peshawar hat er einen Onkel, der wiederum einen Sohn namens Enayatullah hat. Gemeinsam mit seinem Vater betreibt Enayatullah einen Marktstand, an welchem Technikgeräte verkauft werden. Im Gegensatz zu Jamal spricht Enayatullah kein Englisch. Sein Vater wünscht sich mehr für seinen Sohn, als die hoffnungslose Perspektive, die Peshawar ihm bietet. Also sieht er seine Zukunft in London, wo bereits ein Bekannter der Familie lebt. Für viel Geld kauft er Jamal und Enayatullah bei Menschenschmugglern ein Ticket nach London.

Da Jamal der englischen Sprache mächtig ist, soll er Enayatullah bei seiner Reise begleiten. Enayatullah selbst ist älter als Jamal, wirkt im Film eher zurückhaltend und ist den Fluchthelfern gegenüber stets misstrauisch. Dies teilt er Jamal während der Reise immer wieder mit.

Auf der Überfahrt von der Türkei nach Italien erstickt Enayatullah im Container. Am Ende hat es nur Jamal nach London geschafft. Erst hier kann er sich bei Eneyatullah's Vater telefonisch melden, und ihm vom Tod seines Sohnes berichten. Die Beziehung der beiden Cousins zueinander ist recht uneindeutig. Als Zuschauer spürt man weder eine besondere Verbindung, noch eine eindeutige Abneigung.

Durch die außergewöhnliche Fluchtsituation sind Jamal und Enayatullah aufeinander angewiesen und im Laufe des Films geraten sie dadurch oft aneinander. Dennoch sind es auch herzliche Momente, die geteilt und ausgelebt werden: abends im Bett erzählen sie sich Witze und Geschichten, sie lachen gemeinsam und die zum Teil gefährlichen Erlebnisse schweifen sie zusammen. Entsprechend verzweifelt ist Jamal, als er im Container in Italien zur Besinnung kommt und feststellt, dass sein Cousin gestorben ist. Erst, als er sein Ziel in London erreicht hat, kann er seiner Trauer freien Lauf lassen und beginnt, die Eindrücke und Verluste seiner monatelangen Reise zu verarbeiten. 


\subsection{Bildgestaltung, Filmsprache}

\section{Struktur: Dramaturgie, Erzählperspektive(n), Gestaltungs- mittel}

Der Film "In This World" - Aufbruch ins Ungewisse verläuft nach einem klassischen Dramenaufbau, so dass eine typische Verlaufskurve der Veranschaulichung dienen kann.

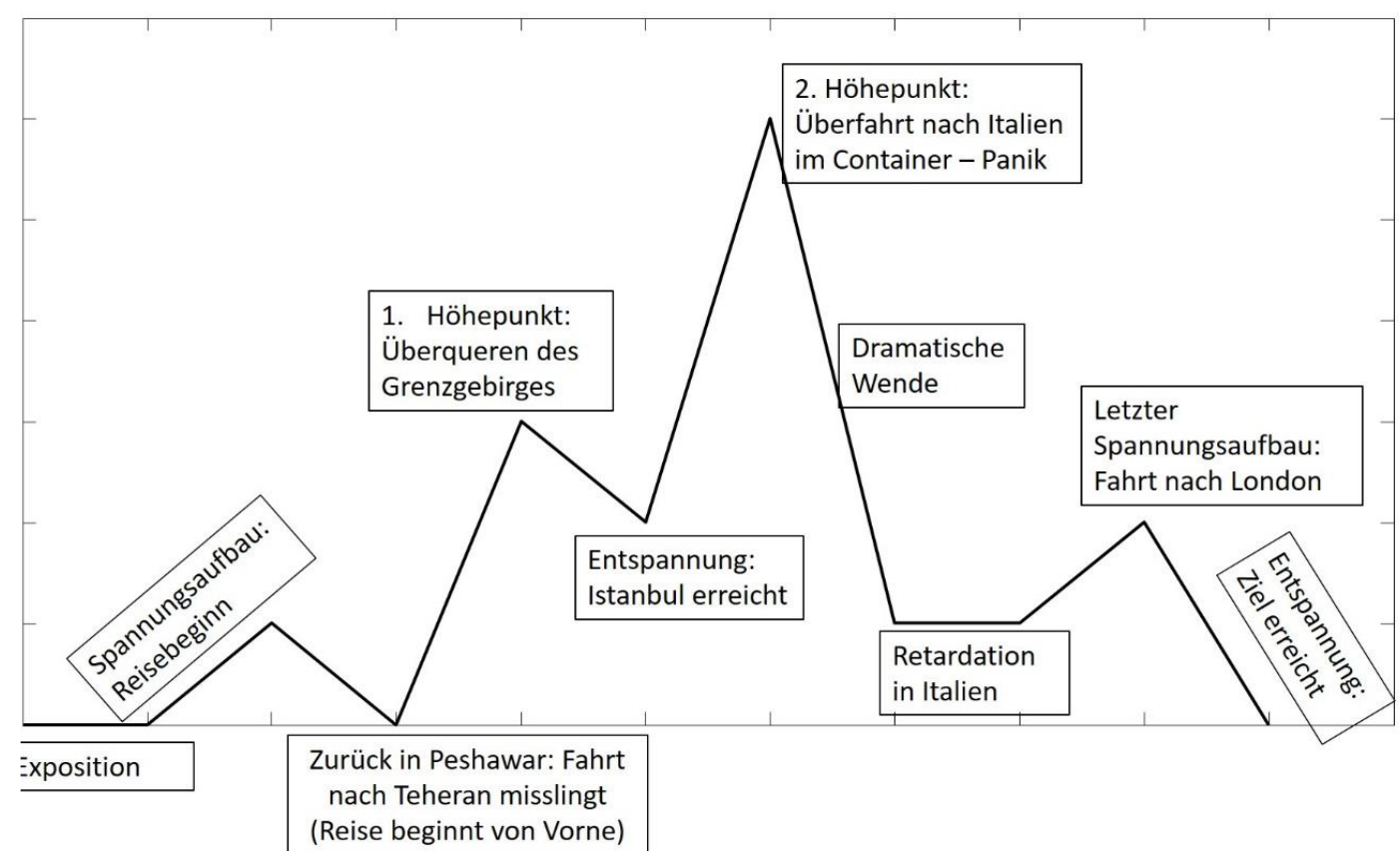

Abbildung 7: Dramenverlaufskurve für den Film „In This World”.

Die Einleitung erfolgt im Film mit der Vorstellung des Flüchtlingslagers Shamshatoo in Pakistan. Durch das Voice-Over erhält der Zuschauer erste Informationen über den Ort des Geschehens und die derzeitige soziale Situation, sowie historisch-politische Aspekte, die zu dieser geführt haben. Zusätzliche Informationen wie Orts- bzw. Staatsnamen und Zeitpunkte werden im Film schriftlich eingeblendet.

Des Weiteren werden in der Exposition des Filmes die Hauptcharaktere vorgestellt. Mit ersten Einblicken in ihr Leben steigt der Zuschauer in das weiterlaufende Geschehen ein und begleitet Jamal und Enayatullah von da an bis zum Ende des Films. Mit dem Kauf eines Fluchttickets von Pakistan nach London bei korrupten Menschenschmugglern ist die Thematik des Films festgelegt, und der Zuschauer erkennt, dass er Jamal und Enayatullah auf ihrer 
Flucht begleiten darf. Die Exposition endet mit dem Beginn ihrer Reise.

Von da an steigert sich die Handlung kontinuierlich und mit jeder neuen Etappe, die die beiden erreichen, kommen sie dem Spannungshöhepunkt näher. Der Zuschauer bangt bei jeder Weiter- oder Überfahrt, bei jedem neuen Schmuggler, dem keine eindeutig positiven Absichten zugesprochen werden und bei jedem Aufeinandertreffen mit Grenzsoldaten oder Polizeibeamten um das Gelingen der Flucht.

Abgebaut wird der Spannungsbogen, als die beiden Cousins bei einer Kontrolle im Iran auf dem Weg nach Teheran angehalten und später, aufgrund fehlender Ausweispapiere, in der Wüste ausgesetzt werden.

Schließlich landen sie wieder dort, wo ihre Reise ursprünglich begann. Ab hier muss der Spannungsbogen erneut aufgebaut werden, und verläuft mit dem zweiten, ungewissen Versuch umso steiler. Je mehr sich Jamal und Enayatullah von Pakistan entfernen und je näher sie ihrem Ziel kommen, desto gefährlicher scheint ihre Flucht zu werden.

Besonders nervenaufreibend ist das Überqueren des tief verschneiten Grenzgebirges, das den Iran von der Türkei trennt. Grenzbeamte suchen das Gebiet auf illegale Flüchtlinge $a b$, und sind bereit, diese zu erschießen. Um das Risiko einer solchen Begegnung zu vermindern, reisen Jamal und Enayatullah in der Nacht, wodurch der Weg umso schwieriger wird. Dann hören sie Schüsse und Schreie. Die Dunkelheit gibt keine Auskunft, ob in diesem Moment Menschen zu Tode kommen. Panisch verstecken sie sich - die Spannung erreicht einen ersten Höhepunkt: schaffen es Jamal und Enayatullah lebend in die Türkei?

Am nächsten Morgen sieht man sie auf der Ladefläche eines Pickups, der sie nach Istanbul fährt. Hier sinkt die Kurve zunächst wieder, die Situation entspannt sich. In Istanbul haben die beiden Jungen Zeit, sich von den Strapazen zu erholen. Man sieht sie Eis essen und in einer Werkstatt arbeiten. Sie finden Menschen, denen es ähnlich ergeht, wie ihnen, und die denselben Fluchtweg teilen. Mit den Kindern spielen sie Fußball und sind sichtlich froh darüber, dass sie den Weg über das Gebirge geschafft haben. 
Erst, als sie und andere Flüchtlinge von den nächsten Fluchthelfern abgeholt werden, steigt der Spannungsbogen wieder. In einem Container eingeschlossen verbringen sie viele Stunden auf der Ladefläche eines LKW's, welcher auf ein Schiff verfrachtet wurde. In völliger Dunkelheit und ausgestattet mit nur einer Taschenlampe hört man ununterbrochen das Schreien eines Babys, das mit seinen Eltern nach Dänemark flüchtet.

Durch die nervenaufreibende Situation, der sich die Menschen im Container nicht entziehen können, wird die Spannung immer gröBer. Die Flüchtlinge merken, dass ihnen die Luft ausgeht. Dann bricht Panik aus. Verzweifelt klopfen sie an die Wände des Containers, schreien, wollen herausgeholt werden, doch keiner kann sie hören.

40 Stunden später wird der Container geöffnet. Bis auf Jamal und das Baby, das weinend im Arm seiner toten Mutter liegt, sind alle Insassen während der Überfahrt nach Italien erstickt. Hier nimmt der Film eine traurige und dramatische Wende - nicht nur für Jamal, der von nun an ohne seinen Cousin weiterreisen muss, sondern auch für das Baby, dessen Zukunft ohne seine Eltern ungewiss ist.

Durch die hoffnungslos scheinende Situation, der Jamal von nun an ohne Enayatullah und ohne Geld in einem ihm völlig fremden Land ausgesetzt ist, fällt die Handlung weiter. Die Retardation obliegt dem Diebstahl einer Handtasche, von dessen Inhalt sich Jamal ein Ticket nach Paris kauft.

Im Flüchtlingslager in Sangatte, an der nördlichen Küste Frankreichs, ist das Ziel bereits zum Greifen nahe. Mit der nicht ganz ungefährlichen Weiterfahrt, zu der sich Jamal schließlich mit einem neugewonnenen Kumpan entscheidet, steigt die Handlungsspannung ein letztes Mal. Doch da diese letzte Etappe recht unkompliziert erreicht wird, baut der Bogen sich erneut ab. Mit der dann eingeblendeten Szene von Jamal, der in einem Restaurant in London arbeitet, scheint der Konflikt gelöst und das Fluchtziel erreicht.

Der Film endet in einer Moschee, wo Jamal seinen Tränen freien Lauf lässt und das Erlebte betend verarbeitet. Ein Voice-Over 
schließt den Film ab. Der Sprecher erzählt, welche durchaus verschiedenen Wege Jamal und Enayatullah nach Abschluss der Dreharbeiten gegangen sind.

Die Erzählperspektive im Film ist grundsätzlich die eines neutralen Erzählers, der das Geschehen weder kommentiert noch aus der Perspektive eines bestimmten Charakters heraus erzählt. Die Handlung wird vom Zuschauer nur durch die Registrierung des Äußeren und die dabei anfallenden Dialoge der Charaktere erschlossen. In der Exposition sowie am Ende des Films tritt eine Erzählstimme, ein männliches Voice Over, in akustische Erscheinung, die den Zuschauer über sachliches Hintergrundwissen der Darsteller, der Orte und sozialhistorischer Aspekte informiert.

Die Struktur und der Aufbau des Films passen sich der Fluchtstrecke der beiden Jungen an. Etappenweise kommen Jamal und Enayatullah ihrem Ziel immer näher, und jeder neu erreichte Ort oder jeder erfolgreiche Staatswechsel obliegt den Impressionen, mit deren Hilfe diese Umgebunden zunächst vorgestellt werden, so dass die Zuschauer wissen, wo sich die Cousins gerade befinden.

Ein besonders hilfreiches Gestaltungsmittel ist die animierte, politische Karte, die immer wieder eingeblendet wird. Eine rote Linie kennzeichnet dabei den Weg, den Jamal und Enayatullah hinter sich bringen und können dem Zuschauer dadurch eine zusätzliche Orientierung geben. Unterstützend sind dabei auch schriftlich eingeblendete Orts- oder Staatsnamen bzw. zeitliche Angaben, die deutlich machen, wie lange eine solche Reise dauert.

Nach den Impressionen zu bestimmten Orten und Umgebungen, wo sich die Jungs in der Regel einige Tage aufhalten, folgen oft Szenen vom dortigen Alltag, in den auch Jamal und Enayatullah eingebunden werden. Und so kommt es immer wieder zu Szenen, in denen sie ausgelassen mit einheimischen Kindern Fußball spielen, durch die Gassen schlendern, beten, sich waschen, oder sich im Bett Geschichten erzählen. Befinden sie sich gerade auf dem Weg zum nächsten Ortsziel, so werden auch diese Wege eingeblendet: Gut ausgebaute Hauptstraßen, auf denen sie mit Bussen oder auf den Ladeflächen kleinerer Laster fahren, holprige Wege 
auf trockenem Wüstenboden, oder mit tiefem Schnee bedeckte Gebirge, die überquert werden müssen.

Auf diese Weise ist der Film durch eine immer wiederkehrende Struktur gestaltet: Auf Impressionen der Umgebung folgen Szenen des Alltags, in welchem Jamal und Enayatullah eingebunden werden. Zwischen den Umgebunden werden Wege und Verkehrsmittel gezeigt, mit denen sie reisen. Jedes neu erreichte Ziel wird durch eine schriftliche Einblende oder mit Hilfe der politischen Karte gekennzeichnet. 


\section{Technik und Filmsprache}

\begin{tabular}{|l|l|}
\hline Sequenz & Inhalt \\
\hline Sequenz 2 & $\begin{array}{l}\text { Die Einleitung des Films beginnt mit dem Flüchtlingslager in Shams- } \\
\text { hatoo: Bilder von Kindern werden schnell und sprunghaft aneinan- } \\
\text { dergereiht, was die traurige Situation vor Ort deutlich macht, näm- } \\
\text { lich der Hoffnungs- und Perspektivlosigkeit vieler kleiner Kinder, die } \\
\text { ihr Leben noch vor sich haben. } \\
\text { Und dennoch: Die Kinder schauen fröhlich in die Kamera, lachen } \\
\text { oder spielen. Sie sind sich ihres Schicksals nicht bewusst und ver- } \\
\text { bringen die Tage sorglos mit den Möglichkeiten, die ihnen zur Ver- } \\
\text { fügung stehen und mit denen sie aufgewachsen sind. Auf der ande- } \\
\text { ren Seite müssen ältere Kinder schon früh Verantwortung überneh- } \\
\text { men: Jamal wird beim Arbeiten in der Steinfabrik gezeigt. Die Auf- } \\
\text { nahmen zeigen ihn von hinten. Es wird dem Zuschauer nicht er- } \\
\text { möglicht, ihn über seine Arbeit zu identifizieren, womit man davon } \\
\text { ausgehen kann, dass er diese Tätigkeit nicht aus einem freien Willen } \\
\text { heraus verübt. } \\
\text { Während weitere Bilder von kleinen Kindern eingeblendet werden, } \\
\text { erfahren wir vom Sprecher, dass 2001 7,9 Milliarden Dollar für die } \\
\text { Bombardierung Afghanistans ausgegeben wurden. Die Kombination }\end{array}$ \\
\hline
\end{tabular}




\begin{tabular}{|l|l|}
\hline & von Information und Bild ist bewusst gesetzt, denn es zeigt die fol- \\
gewidrigen Konsequenzen sinnloser Kriegsführungen, in denen un- \\
zählige Unschuldige zu Tode kommen oder vertrieben werden, da- \\
runter auch Kinder. Kurz nach dieser Information folgt eine Szene, \\
in welcher ein Kind über ein karges, endlos scheinendes Feld läuft, \\
so dass eine Ausweglosigkeit verdeutlicht wird. \\
Das Voice-Over, über das der Zuschauer wichtige Hintergrundinfor- \\
mationen erhält, lässt den Film wie einen Dokumentarfilm wirken. \\
Im Laufe des Films bleibt diese Annahme erhalten. Der neutrale Er- \\
zähler berichtet nur bildlich über das äußerlich Registrierte und be- \\
gleitet die beiden Jungen bei ihrer Reise auf dokumentarische \\
Weise, als wäre er selbst ein Flüchtender. Dadurch gewinnt der Film \\
an Authentizität, so dass eine tiefgreifende Message der Realität \\
versendet wird: Das, was im Film passiert, passiert jeden Tag auf \\
dieser Welt, wir wissen es, aber sehen es nicht - also macht der \\
Film es sichtbar!
\end{tabular}




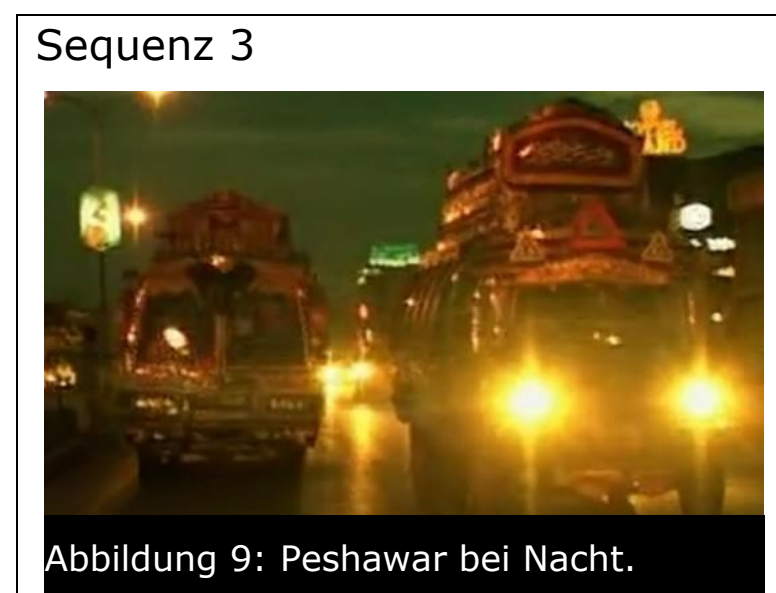

http://medienwelten.mp.ew.tu-dresden.de
In der Flüchtlingsmetropole Peshawar herrscht Hektik. Die schnelle Aneinanderreihung von Eindrücken ist inhaltslos, jedoch bedeutsam für eben diese hektische Eigenschaft der Großstadt. Anders als in Shamshatoo fahren hier viele Autos durch die Straßen. Die verschiedenen Aufnahmen von Fahrzeugen verstärken die städtische Lebendigkeit.

Durch die Dunkelheit des Abends und das Leuchten der Autos und Häuser kann der Zuschauer eine zeitliche Einordnung vornehmen: Es ist so spät, dass die Dunkelheit die Stadt erreicht hat, aber noch zu früh für den nächtlichen Schlaf ihrer Bewohner. Fröhliche, orientalische Musik lässt die Stadt lebendig wirken. Es scheint angenehm kühl, aufregend und unbeschwert, so dass Peshawar im starken Kontrast zum heißen, trostlosen und unbewegten Shamshatoo steht. Und trotz der durchaus besseren Möglichkeiten, die eine Großstadt wie Peshawar den Menschen bietet, scheint es auch hier für Afghanen ein trostloser, 21 perspektivloser Ort zu sein. Szenen von frei fliegenden Vögeln am Himmeln sind im starken Kontrast zu den dort lebenden Menschen, die der Stadt zu gehören scheinen. 

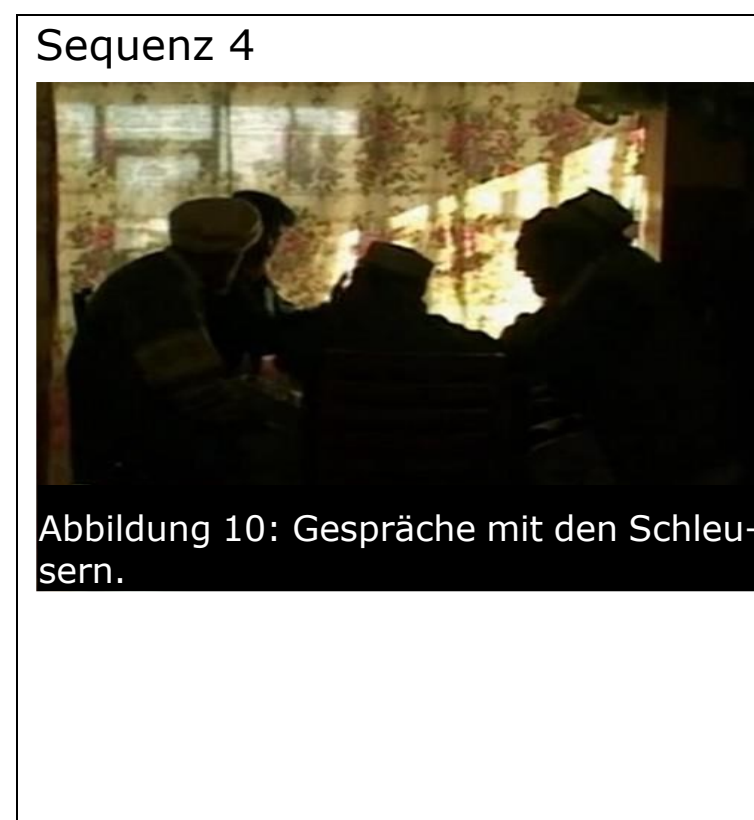

Sequenz 8
Die Fluchtgespräche mit dem Schleuser sind im vollen Gange. Die Kamera filmt die Gespräche aus halbnaher Distanz, wodurch die Verhandlung als etwas sehr Intimes, Geheimes oder Verbotenes wirkt. Auf diese Weise wird die dokumentarische Authentizität des Films zusätzlich gesteigert, denn es scheint, als würden die Aufnahmen heimlich gefilmt werden.

Durch den abgedunkelten Raum und Jamal, der mit dem Rücken zur Kamera sitzt, so dass der Zuschauer vom Gespräch ausgeschlossen wird, erhält die Situation eine gewisse Kriminalität: Hier wird Verbotenes, etwas zu Schützendes besprochen. Nur die Dialogausschnitte geben Auskunft über das Gesagte. Die Männer verhandeln über die Flucht der beiden Jungen, die mit Hilfe eines illegalen Netzwerkes aus Menschenschmugglern ermöglicht werden soll.

Jamal und Enayatullah nehmen Abschied. Langsame, einfühlsame Musik betont die emotionale Situation und reflektiert sie auch auf den Zuschauer. Es ist dunkel und die Personen werden nicht zusätzlich ausgeleuchtet, womit eine gewisse Angst vor dem Unbekannten symbolisiert werden soll. Zudem werden die Abschiednehmenden nah erfasst, so dass man ihre Emotionen deutlich anhand ihrer Gesichtszüge erkennen kann. An dieser Stelle wird dem Zuschauer ein Einblick in den intimen und sehr persönlichen Moment gewährt. Man 


\begin{tabular}{|l|l|}
\hline & $\begin{array}{l}\text { selbst wird eingeladen, am Abschied teilzuhaben. Dann filmt die Ka- } \\
\text { mera abwechselnd aus Sicht der Zurückbleibenden und der Flüch- } \\
\text { tenden: der Bus fährt los und entzweit sie voneinander. Die zuneh- } \\
\text { mende und endgültige Entfernung wird spürbar und der Zuschauer } \\
\text { kann sich auf diese Weise sowohl in die Gefühlslage von Jamal und } \\
\text { Enayatullah, als auch in die ihrer Familie hineinversetzten. }\end{array}$ \\
\hline Sequenz 9 & $\begin{array}{l}\text { Eine animierte, politische Karte zeigt Peshawar als Startpunkt der } \\
\text { Reise. Von da an folgt eine rote Linie der Route, die die beiden } \\
\text { Cousins zurücklegen. Dadurch gewinnt der Film zusätzlich an Au- } \\
\text { thentizität, da die Karte als eine realistische Darstellung des Flucht- } \\
\text { weges fungiert. Um das dramatische Problem flüchtender Men- } \\
\text { schen, das thematisiert wird, zu betonen, informiert das Voice-Over } \\
\text { den Zuschauer parallel zur Grafik über sachliches Hintergrundwis- } \\
\text { sen: "14,5 Millionen Flüchtlinge gibt es auf dieser Welt. Jedes Jahr } \\
\text { begeben sich etwa 1 Millionen Flüchtlinge in die Hände von Schleu- } \\
\text { sern. Nicht alle kommen in ihren Zielländern an. Viele werden un- } \\
\text { terwegs festgenommen und nicht wenige sterben." } \\
\text { Abbildung 11: Die Karte fungiert immenter } \\
\text { wieder als roter Faden der Fluchtroute. } \\
\text { Durch die animierte Weltkugel wird in Verbindung mit dieser Infor- } \\
\text { mation ersichtlich, dass das Problem auf der ganzen Welt präsent } \\
\text { ist und uns alle betrifft. Zudem wird die Spannung durch das vom } \\
\text { Sprecher Gesagte aufgebaut, denn: nicht alle Schaffen die Flucht }\end{array}$ \\
\hline
\end{tabular}




\begin{tabular}{|l|l|}
\hline & $\begin{array}{l}\text { erfolgreich. Was passiert also mit Jamal und Enayatullah? Werden } \\
\text { sie es nach London schaffen? }\end{array}$ \\
\hline Sequenz 10 & $\begin{array}{l}\text { In Quetta lassen Kinder selbstgebaute Papierdrachen steigen. Die } \\
\text { Kamera filmt gen Himmel. Die in ihrer Perspektivlosigkeit gefange- } \\
\text { nen Kindern blicken nach oben. Ein freies, selbstbestimmtes Leben } \\
\text { scheint unerreichbar. Und doch sind die Drachen auch nicht absolut } \\
\text { frei: an Schnüren befestigt sind sie in den Händen der Kinder ge- } \\
\text { fangen. Somit können sie als Symbol ihrer selbst dargestellt wer- } \\
\text { den: Innerhalb ihrer Grenzen können sie sich frei bewegen, doch } \\
\text { darüber hinaus wird ihnen keine Aussicht auf eine Zukunft in Frei- } \\
\text { heit gegeben. } \\
\text { Abbildung 12: Freude und Gelassenheit } \\
\text { trotz der Fluchtumstände bei Jamal. }\end{array}$ \\
$\begin{array}{l}\text { Abends liegen Jamal und Enayatullah in ihren Betten und erzählen } \\
\text { sich Geschichten. Die Kameraeinstellung filmt ihre Gesichter nah. } \\
\text { Sie sind beleuchtet. Es wird eine gewisse Intimität und durch die } \\
\text { Beleuchtung eine Wärme zwischen den beiden ausgestrahlt. Am } \\
\text { nächsten Morgen schauen die Jungen ins Tal hinab und genießen } \\
\text { die Aussicht der Stadt. Die Kamera filmt die beiden von hinten, so } \\
\text { dass der Zuschauer in den Genuss kommt, diesen Moment mit Ja- } \\
\text { mal und Enayatullah gemeinsam zu erleben. So fühlt man sich den } \\
\text { beiden Nahe und dazugehörig. }\end{array}$ \\
\hline
\end{tabular}




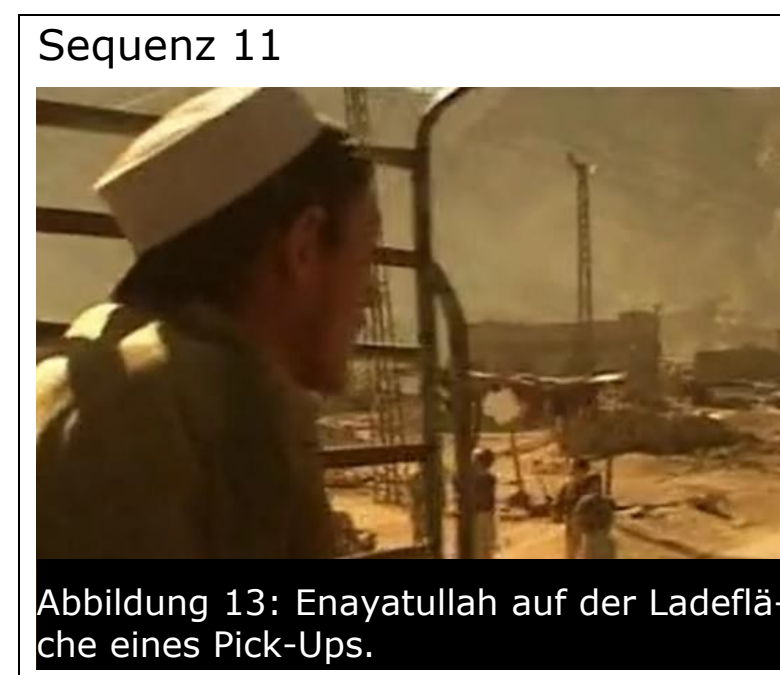

Sequenz 12
Auf dem Weg zum nächsten Treffpunkt wird ihre Reise auf mehreren Autos, Lastern oder Pickups dokumentiert. Die Kamera filmt die Fahrzeuge aus verschiedenen Perspektiven. Meistens wird die Fahrt aus Sicht der Jungen von den Ladeflächen der Autos gefilmt, wodurch eine Nähe zu den beiden hergestellt wird, so dass man sich den Flüchtenden zugehörig fühlt. Dann spürt der Zuschauer auch den holprigen Boden, der durch das ruckartige Wackeln der Kamera ersichtlich wird. Oft aber werden die Fahrzeuge auch von vorne oder hinten gezeigt, wodurch das langsame Vorankommen gezeigt wird. Oder aber die Kamera filmt aus einer unbewegten Perspektive, wie die Auto vorbeifahren, so dass ein Stückweit die Trägheit spürbar wird, mit welcher sich diese fortbewegen zu versuchen. Durch die Aneinanderreihung verschiedener Perspektiven bezogen auf die Fahrzeuge wird die Vielzahl an Etappen und die Langatmigkeit der Erreichung dieser betont. Das setzt sich im Filmverlauf kontinuierlich fort.

Jamal und Enayatullah streiten auf der Ladefläche des Pickups. Die Kamera filmt inre Gesichter abwechselnd nah, je nachdem, wer gerade das Wort ergreift. Auf diese Weise kann dem Streitgespräch gut gefolgt werden. Die Gesichter wirken gestresst und entnervt, der Zuschauer ist durch diese Kameraeinstellung Zeuge des privaten Streits und wieder fühlt man sich als ein dritter, dazugehöriger Flüchtling. Kurze Zeit später machen sie eine Pause.Die Kamera 


\begin{tabular}{|l|l|}
\hline Sequenz 13 & $\begin{array}{l}\text { filmt die beiden aus einer entfernten Perspektive. Die Konsequen- } \\
\text { zen des Streits werden deutlich, der Konflikt ist noch nicht gelöst - } \\
\text { das zeigt die Distanz der Jungen zueinander, die durch die entfernte } \\
\text { Kameraeinstellung zusätzlich verdeutlicht wird. }\end{array}$ \\
\hline $\begin{array}{l}\text { Abbildung 14: Jamal und Enayatullah } \\
\text { bei einem Fluchthelfer. }\end{array}$ & $\begin{array}{l}\text { Sie treffen auf den nächsten Fluchthelfer. Generell werden solche } \\
\text { Gespräche von einer distanzierten Perspektive gefilmt, da sie somit } \\
\text { privat, intim und gefährlich wirken. Dabei versteckt die Kamera sich } \\
\text { oft hinter Vorhängen oder Zäunen, so dass der Eindruck entsteht, } \\
\text { dass heimlich gefilmt wird. Auch die Dunkelheit der Räume ist ein } \\
\text { Symbol für das Geheimnisvolle der Gespräche, das durch diese } \\
\text { Filmgestaltung umso deutlicher wird. Durch die beiden Jungen, die } \\
\text { mit dem Rücken zur Kamera sitzen, schließt sich der Gesprächs- } \\
\text { kreis. Dadurch werden die Zuschauer bewusst vom Gespräch aus- } \\
\text { geschlossen. Diese Mittel werden oft auch beim Telefonieren mit } \\
\text { ihren in Peshawar zurückgebliebenen Verwandten verwendet, um } \\
\text { den beiden Jungen die nötige Privatsphäre zu lassen, die anstands- } \\
\text { halber in unseren Gesellschaften üblich ist. }\end{array}$ \\
\hline Sequenz 14 & $\begin{array}{l}\text { Jamal und Enayatullah machen sich in einem Auto gemeinsam mit } \\
\text { ihrem Schleuser auf den Weg zum Bus, der sie nach Teheran fahren } \\
\text { soll. Hier wird der Schleuser aus einer nahen Perspektive heraus } \\
\text { gefilmt, während er klare Anweisungen gibt, wie sich die beiden im }\end{array}$ \\
\hline
\end{tabular}




\begin{tabular}{|c|c|}
\hline & $\begin{array}{l}\text { Falle einer Kontrolle zu verhalten haben. Dadurch wird dem Zu- } \\
\text { schauer die Wichtigkeit dieser Informationen bewusst, so dass die } \\
\text { Worte des Schleusers als sehr ernstzunehmend erscheinen. }\end{array}$ \\
\hline Sequenz 15 & $\begin{array}{l}\text { Im Bus nach Teheran werden sie vom Militär kontrolliert. Die Ka- } \\
\text { mera filmt aus Sichthöhe der Soldaten zu Jamal und Enayatullah } \\
\text { hinunter. Diese Froschperspektive macht die Unterlegenheit der } \\
\text { beiden Jungen deutlich, auf der anderen Seite zeigt sie die macht- } \\
\text { volle Position und die damit verbundene Überlegenheit, mit der die } \\
\text { Beamten den Menschen im Bus begegnen. }\end{array}$ \\
\hline \multicolumn{2}{|c|}{$\begin{array}{l}\text { Abbildung 15: Perspektive der Gren- } \\
\text { zer auf die Protagonisten. }\end{array}$} \\
\hline Sequenz 16 & $\begin{array}{l}\text { In der Wüste werden sie von den Soldaten ausgesetzt. Die Kamera } \\
\text { ist weit von Jamal und Enayatullah entfernt, so dass die unendlich } \\
\text { Weite der Wüstenlandschaft erkennbar wird, durch die sie sich zu } \\
\text { Fuß ohne Anhaltspunkte und Hilfe durchzukämpfen haben. }\end{array}$ \\
\hline Sequenz 18 & $\begin{array}{l}\text { In Teheran folgen Impressionen der Hauptstadt, die dem Zuschauer } \\
\text { einen ersten Eindruck bieten. Besonders häufig werden Bilder von } \\
\text { modernen Frauen gezeigt, die den Kontrast zu den ärmlichen Ver- } \\
\text { hältnissen auf dem Land verstärken. Auch hier wird durch die }\end{array}$ \\
\hline
\end{tabular}




\begin{tabular}{|c|c|}
\hline & $\begin{array}{l}\text { schnelle Aneinanderreihung von Szenen das Großstadtleben ver- } \\
\text { deutlicht. }\end{array}$ \\
\hline $\begin{array}{l}\text { Abbildung 16: Bei N } \\
\text { Protagonisten im Sch }\end{array}$ & $\begin{array}{l}\text { Jamal und Enayatullah machen sich zu Fuß auf den Weg über das } \\
\text { verschneite Grenzgebirge, das überquert werden muss, um in die } \\
\text { Türkei zu gelangen. Um nicht gesehen zu werden, sind sie nachts } \\
\text { unterwegs. Eine Nachtsichtkamera macht deutlich, wie beschwer- } \\
\text { lich der Weg durch den tiefen Schnee ist, und wie unklar die Umge- } \\
\text { bung zu erkennen ist. Die Kälte der Nacht wird durch } 24 \text { die Geräu- } \\
\text { sche des Windes spürbar. Zwischendurch ist das schwere Atmen } \\
\text { und Schnaufen der Jungen zu hören, die völlig erschöpft von den } \\
\text { Strapazen dieser Route gezwungen sind, den Weg fortzusetzen. Von } \\
\text { weiten werden die drei Jungen mit einer einfachen Handkamera in } \\
\text { der Dunkelheit gefilmt. Dabei werden lediglich die schwarzen } \\
\text { Punkte ihrer Körper vor der hellen Schneekulisse ersichtlich. Die } \\
\text { Aufnahmen sind abgehakt, womit der langsame und mühsame Weg } \\
\text { dargestellt wird. } \\
\text { Die Kamera kann nicht alle Bewegungen flüssig erfassen, eine in } \\
\text { der Nacht typische Orientierungslosigkeit kommt zur Geltung. } \\
\text { Durch die Dunkelheit kann die Kamera die Geschehnisse nur ober- } \\
\text { flächlich und undeutlich erfassen. Die Angst und die Gefahr vor dem } \\
\text { Unbekannten wird übermittelt. Dann sind zwei helle Lichter in der } \\
\text { schwarz-weiß Aufnahme zu erkennen. Sie zeigen, dass die Jungs }\end{array}$ \\
\hline
\end{tabular}




\begin{tabular}{|l|l|}
\hline & $\begin{array}{l}\text { nicht alleine sind. Plötzlich sind Schüsse zu hören. Jamal und Enaya- } \\
\text { tullah wissen, dass diese in Verbindung mit den Lichtern stehen. Sie } \\
\text { nutzen die Dunkelheit der Nacht, um sich zu verstecken. Es wird } \\
\text { immer dunkler, man kann sie nicht mehr sehen }\end{array}$ \\
\hline Sequenz 24 & $\begin{array}{l}\text { Szenen von sexuell angehauchten Musikvideos werden gezeigt, } \\
\text { wodurch dem Zuschauer bewusstwird, dass die beiden Jungen in } \\
\text { der modernen Hauptstadt der Türkei angekommen sind. Dann wer- } \\
\text { den Jamal und Enayatullah von oben gefilmt. An dieser Stelle wird } \\
\text { eine gewisse Verlegenheit ausgedrückt: die beiden Jungen sind den } \\
\text { freizügigen Bildern im Fernsehen unterworfen. }\end{array}$ \\
\hline Sequenz 27 & $\begin{array}{l}\text { Im Container eingeschlossen sollen die Flüchtlinge nach Italien ge- } \\
\text { bracht werden. Der Container ist lichtundurchlässig. Die Dunkelheit } \\
\text { symbolisiert das Unbekannte: Was passiert jetzt? Wo sind wir ge- } \\
\text { rade? Eine Taschenlampe ermöglicht kleine Lichtpunkte auf die ge- } \\
\text { stressten und verängstigten Menschen. Im Hintergrund schreit das } \\
\text { Baby ununterbrochen. Die Flüchtlinge reden durcheinander. Lang- } \\
\text { sam geht den Passagieren die Luft aus und es bricht Panik aus. Die } \\
\text { Panik kann nicht gesehen werden, und dennoch ist sie durch die } \\
\text { Schreie und das Klopfen gegen die Stahlwände des Containers spür- } \\
\text { bar. Die Orientierungslosigkeit im Raum gibt auch dem Zuschauer } \\
\text { das Gefühl einer aussichtlosen Stresssituation, der man nicht ent- } \\
\text { kommen kann. Den Menschen im Raum geht es zunehmend } \\
\text { schlechter. Traurige, ruhige Musik und langsame, langgezogene }\end{array}$ \\
\hline $\begin{array}{l}\text { Abbildung 17: Die letzten Momente von } \\
\text { Enayatullah. }\end{array}$
\end{tabular}




\begin{tabular}{|l|l|}
\hline & $\begin{array}{l}\text { Aufnahmen machen deutlich, dass innen die Kraft ausgeht. Lang- } \\
\text { sam frieren ihre Bewegungen ein. Die Aufnahmen sind abgehakt } \\
\text { und verlangsamt, die Stimmen verstummen. Es ist dunkel: Der Zu- } \\
\text { schauer weiß, dass die Menschen bewusstlos sind, ohne sie zu se- } \\
\text { hen. Die Musik läuft weiter, während der LKW über die Autobahn } \\
\text { fährt. Durch die Musik wird der Zuschauer sensibilisiert und darauf } \\
\text { vorbereitet, dass der Container bald geöffnet wird und dass das zu } \\
\text { einer dramatischen Wende des Films führen wird. }\end{array}$ \\
\hline Sequenz 28 & $\begin{array}{l}\text { Die Musik läuft im Hintergrund weiter und emotionalisiert den Mo- } \\
\text { ment, in dem der Container geöffnet wird und die Mehrzahl der In- } \\
\text { sassen tot aufgefunden werden. Jamal, der neben dem Baby als } \\
\text { einziger überlebt hat, sieht seinen toten Cousin am Boden liegen. } \\
\text { Die Musik ist langsam - und auch Jamals Gedanken sind langsam, } \\
\text { er muss sich sortieren, und braucht Zeit, um zu realisieren, was } \\
\text { passiert ist. Dann rennt Jamal weg. Zunächst wird er von hinten } \\
\text { gefilmt, als würde man ihm nachlaufen. Entsprechend wacklig sind } \\
\text { die Aufnahmen. Dann filmt man ihm aus einer vorderen Perspek- } \\
\text { tive. Gemeinsam mit Jamal entflieht der Zuschauer der Situation, } \\
\text { ohne dem völlig verzweifelten Jungen aus den Augen zu verlieren. } \\
\text { Die Kamera filmt sein Gesicht. Sein leidender Blick emotionalisiert } \\
\text { den Zuschauer. Mitgefühl wird ausgelöst, während man mit Jamal } \\
\text { rennt. }\end{array}$ \\
\hline $\begin{array}{l}\text { Abbildung 18: Nach Ankunft in } \\
\text { Triest rennt Jamal weg. }\end{array}$
\end{tabular}




\begin{tabular}{|l|l|}
\hline Sequenz 29 & $\begin{array}{l}\text { In Triest folgen zunächst Bilder wohlhabender Menschen, an die Ja- } \\
\text { mal versucht, Armbänder zu verkaufen. Hier wird der Kontrast zwi- } \\
\text { schen Arm und Reich besonders deutlich: Während Jamal vor der } \\
\text { Armut flieht, wird inm diese umso bewusster, je näher er dem } \\
\text { Reichtum zu kommen scheint, denn in reichen Ländern ist die } \\
\text { Schere zwischen Arm und Reich besonders groß. Dennoch findet er } \\
\text { viele Menschen, die ihm Geld für seine Bänder geben. Die Kamera } \\
\text { filmt dabei immer aus Jamals Sichthöhe. Es wird sichtbar, dass viele } \\
\text { ihm auf Augenhöhe begegnen. }\end{array}$ \\
\hline Sequenz 31 & $\begin{array}{l}\text { Am Strand in Nordfrankreich spielt Jamal mit den anderen Flücht- } \\
\text { lingen Fußball. Die Kamera folgt den schnellen, abgehackten Bewe- } \\
\text { gungen und lässt den Zuschauer dadurch am Spiel teilhaben. Dann } \\
\text { schwenkt die Kamera aufs offene Meer über, wo auch die Flüchtlinge } \\
\text { hinschauen. Die Weite des Meers scheint unüberwindbar, obwohl } \\
\text { die Flüchtenden ihrem Ziel so Nahe sind. Am Horizont, der das Meer } \\
\text { zu verschlucken scheint, suchen sie nach Anhaltspunkten, die einer } \\
\text { Lösung dienlich sind. }\end{array}$ \\
\hline
\end{tabular}




\begin{tabular}{|l|l|}
\hline Sequenz 33 & $\begin{array}{l}\text { Jamal arbeitet in einem Restaurant in London. Die Kamera filmt inn } \\
\text { von hinten. Er spült Geschirr, aber identifizieren kann er sich mit } \\
\text { der Arbeit nicht, weshalb sein eher unglückliches Gesicht nur durch } \\
\text { die Spiegelung sichtbar wird. Er ruft seinen Onkel in Peshawar an } \\
\text { und berichtet inm vom Tod seines Sohnes. Dann kommt keine Rück- } \\
\text { meldung, sein Onkel antwortet nicht mehr. Zunächst weiß der Zu- } \\
\text { schauer nicht, was los ist. Dann wird das Bild seines Onkels in } \\
\text { Peshawar eingeblendet. Die Zeit scheint stehengeblieben, traurig } \\
\text { blickt Enayatullahs Vater ins Leere. 26 Es folgen Szenen aus Pesha- } \\
\text { war und Shamshatoo. Sie zeigen, dass das Leben für die meisten } \\
\text { Menschen weitergeht, dass alles unverändert ist, und dass Enaya- } \\
\text { tullah's Vater mit dem Schicksal seines Sohnes alleine ist. Im Hin- } \\
\text { tergrund läuft langsame, traurige Musik, die die Gefühle des Vaters } \\
\text { betonen und auch die Umstände der vielen Flüchtlinge, die so hoff- } \\
\text { nungslos und unlösbar scheinen: Enayatullah ist auf seiner Flucht } \\
\text { gestorben, die Flucht ist misslungen, und die Hoffnung gestorben. } \\
\text { dert mit dem Schicksal seines Soh- }\end{array}$ \\
\hline Sequenz 34 & $\begin{array}{l}\text { Das Voice-Over informiert den Zuschauer darüber, was aus Jamal } \\
\text { und Enayatullah nach den Dreharbeiten geworden ist. Dem Zu- } \\
\text { schauer wird ersichtlich, welch unterschiedliche Wege die Menschen } \\
\text { in Armutsverhältnissen gehen können. Auch hier wird der dokumen- } \\
\text { tarische Stil des Films verstärkt. Durch die sachlichen Informationen } \\
\text { wird der Film abgerundet, so dass der Eindruck einer Dokumenta- } \\
\text { tion tatsächlich erhalten bleibt. }\end{array}$ \\
\hline
\end{tabular}


Sequenz 35
Der Abspann läuft mit dem Beten Jamals im Hintergrund. Das Ende bleibt dadurch offen: Was wird aus Jamal? Werden seine Gebete erhört? 


\section{Montage}

Die Aneinanderreihung einzelner Szenen oder ganzer Szenengruppen variiert, dennoch ist über den gesamten Film hinweg eine gewisse Montagestruktur erkennbar. Sowohl sichtbare als auch unsichtbare Montageprinzipien werden angewandt, die in einem immer wieder kehrenden Rhythmus zur Geltung kommen. Sichtbare Schnitte erfolgen oft zwischen einem endenden und einem neubeginnenden Tag, oder etappenweise, um die verschiedenen Orte deutlich voneinander zu trennen.

Durch die beschreibende Montage werden die entsprechenden Schauplätze zunächst vorgestellt, bevor bestimmte Alltagssituationen gezeigt werden. Die oft willkürliche wirkende Aneinanderreihung verschiedener Szenen oder Motive bewirkt, dass thematische Schwerpunkte ineinander überfließen, wodurch der Zuschauer sich immer wieder aufs Neue zurechtfinden muss. Eine solch schnelle Montage symbolisiert allerdings die Hektik und das Chaos, oder aber eine gewisse Anhaltslosigkeit, der Jamal und Enayatullah auf ihrem langen Weg nach London ausgesetzt sind.

Um sich auch als Zuschauer innerhalb der einzelnen Etappen orientieren zu können, werden Orts-, Staats- oder Zeitangaben schriftlich oder mit Hilfe der animierten Karte eingeblendet. Auch das Licht ist für den Zuschauer ein bedeutender Anhaltspunkt, durch welche die Einordnung in bestimmte Tageszeiten auszumachen ist.

In den Hauptstädten der einzelnen Metropole werden insbesondere assoziative bzw. vergleichende Montageprinzipien angewandt, die die Schere zwischen Arm und Reich verdeutlichen. In der Hafenstadt Trieste beispielsweise werden Motive wohlhabender Menschen beim Wein und Kaffee trinken mit denen von Jamal verbunden, der versucht, einfache Straßenwaren an eben diese Klientel zu bringen. Die Straßen Londons hingegen werden mit Motiven in Peshawar verbunden, so dass ein starker Kontrast verschiedener kultureller Lebensweisen ersichtlich wird.

Oft werden auch auf symbolische Montage zurückgegriffen, beispielsweise Motive von Vögeln, die hingegen der Perspektivlosigkeit der Menschen in Peshawar frei sind, und deren Möglichkeit 
unbegrenzt. Die selbstgebauten Drachen iranischer Kinder symbolisieren die Situation der Menschen selbst: An Schnüren werden sie von den Kindern festgehalten, während sie fliegen. Innerhalb ihrer Grenzen können sie sich frei bewegen, doch darüber hinaus ist ihnen die absolute Entfaltung ihrer selbst unmöglich.

\section{Medienpädagogische Analyse}

\subsection{Emotionale Erlebnisqualität - Betroffenheit und Iden- tifikation}

Der Film "In This World" ist für ein jüngeres Publikum kaum geeignet, da er wenig Erlebnisqualität für die bis 14-Jährigen besitzt. Weil Kinder generell eine hohe Bereitschaft zum Miterleben und zur Identifikation haben, könnte ihr "totales" Erleben einiger angstauslösender Situationen (z.B. Grenzgebirgsüberquerung; Containerüberfahrt) sogar bedenklich sein.

Insgesamt bietet der Film ein eher geringes Ausmaß an tatsächlicher Erlebnisintensität und Identifikation für diese Altersgruppe, da für sie die Situationen und die Handlung bzw. die dramaturgische Struktur schwer nachvollziehbar sind. Auch gibt es keine Identifikationsfiguren für Kinder, "Jamal" - als der jüngere Protagonist - ist für unter 14-Jährige kaum geeignet.

Für Jugendliche (14- bis 21-Jährige) wiederum gibt es in diesem Film zwar keine Stars, Idole oder "Charaktere" als wirksame Identifikationsobjekte, dafür aber jugendliche Laiendarsteller in einem authentischen Film, der sowohl zeitliche als auch geographische aktuelle Bezüge zu ihrer Alltagsrealität aufweist.

Dies trifft besonders für geflüchtete Jugendliche zu, aber in einer anderen Form von Betroffenheit auch für "aufnehmende" Jugendliche, die mit Geflüchteten in Kontakt kommen. Und selbst wenn dies nicht der Fall ist, so ist doch die Fluchtthematik omnipräsent und damit für jede und jeden Teil der Alltagsrealität.

Die Identifikation Jugendlicher mit den Figuren wird durch den Einsatz von jugendlichen Laiendarstellern unterschiedlichen Alters mit unterschiedlichen Charaktereigenschaften (vgl. Hauptcharaktere) begünstigt. Im Hinblick auf den in "Darsteller" erwähnten 
Einsatz, den die beiden Hauptdarsteller erbringen mussten, indem sie die für den Film inszenierte Flucht tatsächlich unternahmen, könnte man diskutieren, inwieweit dies zumutbare Bedingungen der Filmteilnahme für die beiden Laiendarsteller gewesen sind.

Weitere filmsprachliche Mittel, die eine Parteilichkeit und Nähe zu den Hauptcharakteren sowie eine Betroffenheit bei den Zuschauenden bewirken, sind:

a. die neutrale Erzählperspektive, die das Geschehen weder kommentiert noch aus der Perspektive eines bestimmten Charakters heraus erzählt. Die Erzählperspektive ist zwar neutral, aber auch eingeschränkt in dem, was sie den Zuschauenden wissen lässt. Grundsätzlich kann man nur das sehen und erfahren, was auch Jamal und Enayatullah sehen können.

b. die Kameraeinstellungen, die Jamal und Enayatullah zum Beispiel bei ihren Gesprächen mit Menschenschmugglern halb nah und von hinten zeigen, was trotz des Gefühls der Ausgeschlossenheit durch die gleiche Blickrichtung von Zuschauenden und Protagonisten auch eine Parteilichkeit hervorruft. Bei Aufnahmen auf Ladeflächen von Autos wird meistens aus Sicht der Jungen in der amerikanischen Einstellung gefilmt, sodass man sich den Flüchtenden zugehörig und wie in der Situation anwesend fühlt.

Sogar die Beschaffenheit der Straße wird durch das Wackeln der Kamera spürbar. In anderen Situationen wie dem Geschichtenerzählen im Bett, sind die Gesichter der Protagonisten aus einer nahen Einstellung aufgenommen, wodurch eine gewisse Intimität entsteht.

c. die Beleuchtung bzw. Lichtverhältnisse. Die Fluchtgespräche zum Beispiel werden in halbdunklen Räumen gefilmt, die Situationen erhalten somit den Anschein von etwas Intimem, Geheimem, Verbotenem oder zu Schützendem, was wiederum die dokumentarische Authentizität des Films steigert. Die Zuschauenden tappen dabei im Dunkeln, genau wie die Protagonisten, die immer wieder neu entscheiden müssen, ob sie den dubiosen Personen vertrauen oder nicht. 
Die Gebirgsüberquerung zwischen dem Iran und der Türkei wird mit einer Nachtsichtkamera begleitet, welche die Umgebung nur erahnen und lediglich die schwarzen Schemen der Körper vor der hellen Schneekulisse ersichtlich werden lässt. Die abgehackten Aufnahmen sowie das schwere Atmen und Schnaufen der Flüchtenden lassen den langsamen und mühsamen Weg spürbar werden. Durch die Dunkelheit kann die Kamera die Geschehnisse nur oberflächlich und undeutlich erfassen und die in der Nacht typische Orientierungslosigkeit macht die Angst und die Gefahr spürbar, der die drei Jungen ausgesetzt sind.

Bei der Überfahrt der Flüchtenden in einem lichtundurchlässigen Container herrscht völlige Dunkelheit, die nur von den gelegentlichen Lichtkegeln einer Taschenlampe unterbrochen wird, die kurze Blicke auf die gestressten und verängstigten Insassen ermöglichen.

Die Dunkelheit symbolisiert das Unbekannte, das Ungewisse, die damit verbundene Orientierungslosigkeit verstärkt auch beim Publikum das Gefühl einer aussichtlosen Stresssituation. Völlige Dunkelheit gepaart mit spannungsintensivierenden Geräuschen wie dem Schreien des Kindes und der anderen Insassen, dem Hämmern gegen Containerwände oder Stille - all das erschafft wirkungsvoll Betroffenheit bei den Zuschauenden. Dadurch, dass man nur das sieht, was auch Jamal und Enayatullah sehen, empfindet man auch ihr Ausgeliefertsein an Menschen und Situationen und die Ungewissheit des Erfolgs ihrer Flucht mit innen.

Weitere stilistische Mittel sind:

a. der Einsatz einer Handkamera, deren wacklige Bilder das Gefühl von Mitrennen oder Mitfahren mit den Protagonisten vermitteln.

b. die illustrierende bzw. emotionalisierende Musik. Zum Beispiel deutet am Ende der Überfahrt im Container eine traurige, ruhige Musik an, dass das Öffnen des Containers zu einer dramatischen Wende des Films führen wird. Ebenso emotionalisiert die Musik die Zuschauenden in der Szene der Benachrichtigung von Enayatullahs Vater vom Tod seines Sohnes und macht sie betroffen. 
c. die Kameraperspektive zum Beispiel wird während der Buskontrolle im Iran unter anderem aus Sichthöhe des Beamten zu Jamal und Enayatullah hinunter gefilmt. Dies bringt die machtvolle Position und die damit verbundene Überlegenheit zum Ausdruck, mit der die Beamten den Menschen im Bus begegnen. Aus Sicht der Jungen wird der Beamte von unten gefilmt. Diese Froschperspektive macht ihre Unterlegenheit deutlich und eine Parteilichkeit der Zuschauenden für die Flüchtenden möglich.

d. Symbole, wie zum Beispiel das Fußballspiel, das entlang der Fluchtroute allerorts gespielt wird und ein Gefühl von Unbeschwertheit auf der ansonsten sehr spannungsreichen Reise aufkommen lässt. Die Identifikation mit den Menschen im Film gleich welcher Herkunft über den Sport als universelle Sprache führt ebenfalls zur Steigerung der Parteilichkeit seitens der Zuschauenden.

Fazit: Eine Betroffenheit von dem Geschehen des Films wird durch eine hohe Identifikation mit den Figuren und eine emotionalisierende Filmsprache erreicht.

\section{Psychische Belastung durch bestimmte Inhalte}

Für Jugendliche mit eigener Fluchterfahrung besteht beim Schauen von "In This World" unseres Erachtens mit hoher Wahrscheinlichkeit die Gefahr der Retraumatisierung, weswegen eine Filmvorführung für ebendiese Jugendlichen aus medienpädagogischen Gründen nicht vertretbar ist.

Die FSK-Empfehlung von 12 Jahren halten wir für sinnvoll, wegen der Verlassenheit der zwei Protagonisten und später von Jamal in fremder Umgebung und einiger angstauslösender (Containerüberfahrt, Gebirgsüberquerung) und trauriger Situationen (Tod der Mehrheit der Containerinsassen). 


\subsection{Die kognitive Erlebnisqualität}

Trotz des dokumentarisch anmutenden Filmstils besitzt „In This World" eine Dramaturgie. Diese folgt einem klassischen Dramenaufbau $^{1}$. Es handelt sich um einen semidokumentarischen Film; einen Spielfilm also, der sich den Anschein eines Dokumentarfilms gibt.

Mit diesem relativ ungewohnten Filmstil lassen sich die Sehgewohnheiten der Jugendlichen herausfordern. Die Verständlichkeit der Geschichte wird aber durch verschiedene Gestaltungsmittel erleichtert. Zum Beispiel durch den Einsatz eines Voice-Overs, das sozialhistorische und politische Hintergrundinformationen liefert, und durch Grafiken (animierte politische Karte, eingeblendete Orts-/Staatsnamen, zeitliche Angaben), die die Fluchtroute visualisieren und geographische Kenntnisse erweitern.

Hilfreich ist auch die Erzählstruktur. Jede Etappe wird gleichermaBen strukturiert: erst wird die Umgebung vorgestellt, Grafiken, Ortsnamen/Zeitangaben geben Orientierung, der Weg zum Zwischenziel und Impressionen vom Ort und den ansässigen Menschen werden in einer beschreibenden Montage gezeigt, dann erst spielt sich die Handlung an der jeweiligen Fluchtstation ab. Die Lichtverhältnisse dienen außerdem als Anhaltspunkt für eine tageszeitliche Einordnung des Geschehens.

Dennoch bedeuten manche Szenen größere kognitive Herausforderungen für das Publikum. Die schnelle Aneinanderreihung von Bildern und Szenen etwa vermittelt zwar wirkungsvoll die Hektik größerer Städte, erschwert aber auch das Zurechtfinden der Zuschauenden in den entsprechenden Situationen. Der Effekt ist, dass sie die gleiche Orientierungslosigkeit wie Jamal und Enayatullah bewältigen müssen.

Im Gegensatz dazu stehen die längeren Aufenthalte an einem Ort, die für etwas kognitive Entspannung von der Informationsfülle sorgen. Ein Gefühl für das langsame Vorankommen der beiden

${ }^{1}$ vgl. Struktur: Dramaturgie, Erzählperspektive(n), Gestaltungsmittel) 
Protagonisten auf ihrer Flucht wird durch die Kameraeinstellungen "weit" und "total" vermittelt.

Eine weitere Herausforderung an die Sehgewohnheiten und die kognitiven Fähigkeiten stellt die fehlende Synchronisierung dar. $\mathrm{Zu}$ hören sind die Originalsprachen und -stimmen der Darsteller, die von einer Off-Stimme übersetzt werden. Dies verlangt von den Zuschauenden hohe Konzentration, um das Gesprochene den jeweiligen Personen zuzuordnen. Gleichzeitig erhöht es das Verständnis dafür, welch weite Reise die Protagonisten durch verschiedene Sprachräume zurücklegen und was für eine geistige Flexibilität innen dieser Umstand abverlangt.

Auch die Personenkonstellationen sind nicht unbedingt leicht zu durchschauen. Jamal und Enayatullah haben trotz ihrer Verwandtschaft ein recht uneindeutiges Verhältnis zueinander. Ob sie sich sympathisch sind oder nicht, bleibt unklar. Nur ihre Verbundenheit durch die gemeinsame Flucht und inr Aufeinander-AngewiesenSein sind nachvollziehbare Indikatoren für ihre Beziehung.

Andere Figuren sind und bleiben sowohl in ihrer Identität als auch ihrer Funktion oft diffus. Es ist eine anspruchsvolle Aufgabe, zu entscheiden, ob sie vertrauenswürdig sind oder nicht. Diese Entscheidung muss sowohl das Publikum als auch Jamal und Enayatullah trotz fehlender Anhaltspunkte immer wieder treffen.

\subsection{Die ästhetische Erlebnisqualität}

Die Annahme, dass der Film „In This World" nicht den Sehgewohnheiten von Jugendlichen entspricht, bedeutet nicht zwangsläufig, dass der Film deshalb nicht von Jugendlichen in seiner Außergewöhnlichkeit akzeptiert werden kann. Besonders der Einsatz von jugendlichen Laiendarstellern erleichtert den Rezipienten den Identifikationsprozess und erhöht zudem noch den Grad ihrer Betroffenheit. Auch in seinen Möglichkeiten zu ästhetischer Bildung ist der Film anders als andere Filme.

Wegen seines semi-dokumentarischen Stils wartet "In This World" weder mit Special Effects noch mit besonderer Ausstattung auf. Im Gegenteil; es werden ausschließlich die spontanen Gegebenheiten vor Ort, also die natürliche Ausstattung genutzt. Dies ist 
sicherlich dem Umstand geschuldet, dass alle Drehorte real sind (mit Ausnahme des nachgebauten Flüchtlingslagers Sangatte) und als solche auch authentisch wirken sollen.

Die künstlerische Gestaltung ordnet sich hier dem Anspruch nach größtmöglicher Authentizität unter. Hierin besteht wiederum die Chance, offene Prozesse und neue Erfahrungen in der ästhetischen Bildung Jugendlicher im Medienbereich zu ermöglichen.

Als dramaturgisches Gestaltungselement bedient sich der Film des Öfteren verschiedener Montageprinzipien. Symbole der Freiheit wie Vögel, Papierdrachen oder Pferde werden gezeigt. Letztere scheinen frei und sind doch festgehalten. Die symbolische Montage verknüpft die Bilder der Papierdrachen oder des wilden Pferdes, das unruhig an seinem Seil hin und her tänzelt, mit Bildern der Menschen Pakistans. Auf diese Weise lässt sich eine Analogie herstellen, die von den Jugendlichen als solche wahrgenommen und gedeutet werden kann.

Die assoziative bzw. vergleichende Montage stellt Bilder wohlhabender Menschen aus dem westlichen Kulturkreis beim Kaffeeund Weintrinken neben Bilder von Jamal beim Straßenverkauf, der versucht, Geld für seine Weiterreise zu verdienen. Die Kluft zwischen Arm und Reich drängt sich den Zuschauenden hier bildlich auf wie an keiner anderen Stelle auf der Fluchtroute. Am Ende des Films werden Szenen aus den Straßen Londons mit Szenen aus Peshawar verbunden. Der starke Kontrast zwischen den Kulturen kommt so wirkungsvoll zur Geltung.

Dieser wird auch durch die Musik im Film unterstützt. Zumeist ist On-Ton Musik zu hören, welche die jeweilige Kultur wiederspiegelt und damit zur (ästhetischen) Bildung der Jugendlichen beiträgt. Außerdem dient sie der dokumentarischen Authentizität des Films. Es gibt aber eben auch einen komponierten Score, der "In This World" als Spielfilm erkennen lässt. 


\subsection{Der Film als potentielles Lernfeld und als Kommunika- tionsangebot}

Obschon Filme eher für Kinder eine Sozialisations- und Erziehungsinstanz darstellen können, bietet sich in Bezug auf "In This World" auch für Jugendliche ein potentielles Lernfeld.

Zum Beispiel, was die Personenkonstellationen und die sozialen Beziehungen anbelangt: Da gibt es auf der einen Seite Jamal und Enayatullah, die ungleichen Cousins, die durch ihre gemeinsame Flucht in hohem Maße aufeinander angewiesen sind, deren emotionale Beziehung allerdings schwer zu ergründen ist. Viel wichtiger in ihrer Situation ist aber, dass sie einander gut ergänzen und abwechselnd die Führung und die Entscheidungen auf der Reise übernehmen.

Unklar bleibt die Beziehung von Jamal zu seinen Mitbewohnenden im Flüchtlingslager Shamshatoo in Pakistan. Diese Unklarheit kann als Diskussionsanstoß darüber dienen, unter welchen Bedingungen Menschen in Flüchtlingslagern leben (müssen), und wie sie ihr Leben dort zum Teil über Generationen hinweg gestalten.

Die Familie, die Jamal und Enayatullah in Istanbul kennenlernen und mit der sie in einem Container nach Triest gebracht werden, stirbt auf der Überfahrt. Nur ihr kleiner Sohn überlebt. Es stellt sich die Frage, was mit ihm passieren wird. Das Schicksal dieser Familie ist ein Anlass dafür, mit Jugendlichen darüber zu diskutieren, was Menschen alles für die Aussicht auf ein besseres Leben riskieren.

Auch auf Seiten der Konflikte und des gezeigten Konfliktverhaltens gibt es einige Diskussionsanstöße für Jugendliche. Was bedeutet es zum Beispiel für Enayatullah, wenn Jamal dessen Walkman verschenkt, den er zu Beginn ihrer Flucht von seiner Familie geschenkt bekommen hat und mit dem er Musik aus seiner Heimat hören konnte?

Diese Entscheidung Jamals war gänzlich pragmatisch, denn sie garantierte innen die Weiterreise, aber für Enayatullah hat sie eine emotional schwerwiegende Bedeutung. Rechtfertigt der Zweck hier die Mittel? Die Antwort ist Schweigen und Nicht-Thematisierung seitens der Protagonisten. 
Eine weitere Konfliktsituation stellt die Passkontrolle im Bus auf dem Weg nach Teheran dar. Es ist eine bedrohliche Situation, die entscheidend für den Fluchtverlauf von Jamal und Enayatullah ist. Die beiden stehen in diesem Konflikt passiv dem Beamten gegenüber, der aktiv und gewaltvoll seine politische Macht ausübt. Er stellt mit seinem ganzen Verhalten die Hierarchie wieder her. Der Konflikt endet mit dem Sieg der politischen Macht.

Und schlussendlich schwebt über dem gesamten Film das konflikthafte und zutiefst hierarchische Verhältnis von westlicher und "orientalischer" Welt. Je weiter die Protagonisten in den Westen kommen, desto "moderner" werden die Menschen, bis die Kluft und die Hierarchie in Europa am deutlichsten ins Auge springen.

Dieser Konflikt ist ein globaler und ein politischer, der sich gewaltvoll inszeniert und Menschen zu Flucht und Migration zwingt. Auch Jamal und Enayatullah lösen diesen Konflikt insofern, als dass sie flüchten und so ihr Schicksal selbst in die Hand nehmen, trotz hohem Risiko für ihr Leben.

Die Diskussion des letztgenannten Konflikts kann direkt mit einer Diskussion über die Werte und Wertorientierungen der jugendlichen Zuschauenden verknüpft werden. Was bedeuten uns unsere Werte wie die Würde des Menschen? Wie weit ist es her mit der Chancengleichheit?

Hilfreich als Grundlage für diese Diskussionen ist, dass der Film durch seine Authentizität ein hohes $M a ß$ an Informationen über die Realität und über andere Kulturen bietet. Er hat damit in jedem Fall eine dialogstiftende Qualität und alles, was er braucht, um Kommunikationsprozesse zu initiieren:

Wirksame Identifikationsfiguren, Glaubwürdigkeit durch die Betroffenheit und die parteiliche Sicht der Jugendlichen, eine herausfordernde und dennoch einfühlsame Dramaturgie, eine anspruchsvolle weil lebensnah gestaltete Handlung, keine normgerechten Verhaltensweisen seitens der Protagonisten, sondern innovative und lebenskünstlerische, die, wegen der Ereignisse, zu denen sie führen, mehr offene Fragen zur Diskussion bieten als moralische Zeigefinger. 
Für eine bessere Diskussionsgrundlage würden sich nach dem Film sozialhistorische Informationen über die Lage der Afghanen in Pakistan und Informationen über die Filmemacher und ihre Motivation für den Film anbieten. Es ist dem Regisseur nämlich ein besonderes Anliegen, bewusst auf die Erklärung einer Fluchtmotivation der Protagonisten zu verzichten.

In der Nachbereitung des Films könnten eigene „Flucht"-motivationen der Jugendlichen gesammelt werden, um die oben angedeuteten Diskussionen anzuregen. Allerdings ergibt sich der Kommunikationsbedarf mit großer Wahrscheinlichkeit auch einfach aus dem Film heraus. 


\section{Literatur}

Kellerhoff, Olaf: Die Rolle des Militärs im politischen System Pakistans. In: Bundeszentrale für politische Bildung (14.05.2010). URL: http://www.bpb.de/apuz/32727/dierolle-des-militaers-im-politischen-system-pakistans? $p=$ all (zuletzt besucht am: 30.08.2017).

Khalatbari, Babak: Afghanistan unter dem Terror der Taliban. In: Bundeszentrale für politische Bildung (15.08.20008). Online im Internet. URL: http://www.bpb.de/internationales/asien/afghanistan/48618/terror-der-taliban (zuletzt besucht am: 30.08.2017).

Kilzer, Annette: In This World. Filmheft. Bonn 2003.

Ladumer, Ulrich: Er ist doch unser Gast. Viele Afghanen sind vor der Taliban geflohen. Trotzdem schlägt ihr Herz für Bin Laden. In: Zeit Online (31.10.2001). URL: http://www.zeit.de/2001/45/Er_ist_doch_unser_Gast/komplettan-sicht (zuletzt besucht am: 30.08.2017).

Ruttig, Thomas: Sowjetischer Abzug aus Afghanistan. In: Bundeszentrale für politische Bildung (13.02.2014). URL: http://www.bpb.de/politik/hintergrund-aktu-

ell/178868/1989-sowjetischer-abzug-aus-afghanistan-1302-2014 (zuletzt besucht am: 30.08.2017).

Winterbottom, Michael: In This World. Aufbruch ins Ungewisse. Tiberius Film: Großbritannien, 2014. 